\title{
Lee-Yang zeros of the antiferromagnetic Ising model
}

\author{
FERENC BENCS $\dagger, \ddagger, \S$, PJOTR BUYS§, LORENZO GUERINI§ and HAN PETERS§ \\ $\dagger$ Alfréd Rényi Institute of Mathematics, Reáltanoda u. 13-15, 1053 Budapest, Hungary \\ $\ddagger$ Department of Mathematics, Central European University, \\ Nádor u. 9, 1051 Budapest, Hungary \\ $\S$ Korteweg de Vries Institute for Mathematics, University of Amsterdam, \\ Science Park 107, 1090GE Amsterdam, The Netherlands \\ (e-mail: ferenc.bencs@gmail.com,pjotr.buys@gmail.com, \\ lorenzo.guerini92@gmail.com,hanpeters77@gmail.com)
}

(Received 11 November 2019 and accepted in revised form 19 February 2021)

\begin{abstract}
We investigate the location of zeros for the partition function of the anti-ferromagnetic Ising model, focusing on the zeros lying on the unit circle. We give a precise characterization for the class of rooted Cayley trees, showing that the zeros are nowhere dense on the most interesting circular arcs. In contrast, we prove that when considering all graphs with a given degree bound, the zeros are dense in a circular sub-arc, implying that Cayley trees are in this sense not extremal. The proofs rely on describing the rational dynamical systems arising when considering ratios of partition functions on recursively defined trees.
\end{abstract}

Key words: Ising model, Lee-Yang zeros, complex dynamics

2020 Mathematics Subject Classification: 05C31, 37F10 (Primary); 82B12 (Secondary)

\section{Introduction}

Partition functions play a central role in statistical physics. The distribution of zeros of the partition functions is instrumental in describing phase changes in a variety of contexts. More recently there has been a second motivation for studying the zeros of partition functions, arising from a computational complexity perspective. Since the 1990s there has been significant interest in whether the values of partition functions can be approximated, up to an arbitrarily small multiplicative error, by a polynomial time algorithm. For graphs of bounded degrees this is known to be the case on open connected subsets of the zero free locus [Bar16, PR17]. In recent work of the last author with Regts [PR19, PR20], the zero free locus was successfully described by first considering a specific subclass of graphs, 
the Cayley trees, for which the location of zeros can be described by studying the iteration properties of a rational function.

A common theme in the papers [PR19, PR20] was that the Cayley trees turned out to be extremal within the larger class of bounded degree graphs, in the sense that a maximal zero free locus for Cayley trees proved to be zero-free in the larger class as well. This observation is the main motivation for our studies here, where we investigate to what extent the extremality of the class of Cayley trees holds for the antiferromagnetic Ising model.

Let $G=(V, E)$ denote a simple graph and let $\lambda, b \in \mathbb{C}$. The partition function of the Ising model $Z_{G}(\lambda, b)$ is defined as

$$
Z_{G}(\lambda)=Z_{G}(\lambda, b)=\sum_{U \subseteq V} \lambda^{|U|} \cdot b^{|\delta(U)|},
$$

where $\delta(U)$ denotes the set of edges with one endpoint in $U$ and one endpoint in $U \backslash V$. In this paper we fix $b>0$ and consider the partition function $Z_{G}(\lambda)$ as a polynomial in $\lambda$. The case $b<1$ is often referred to as the ferromagnetic case, while $b>1$ is referred to as the antiferromagnetic case.

For $d \geq 2$ let $\mathcal{G}_{d+1}$ be the set of all graphs of maximum degree at most $d+1$. Given a set of graphs $\mathcal{H}$, we write

$$
\mathcal{Z}_{\mathcal{H}}=\mathcal{Z}_{\mathcal{H}}(b)=\left\{\lambda: Z_{G}(\lambda)=0 \text { for some } G \in \mathcal{H}\right\} .
$$

When $b<1$, the Lee-Yang circle theorem [LY52a, LY52b] states that for any graph $G$, the zeros of $Z_{G}$ are contained in the unit circle $\mathbb{S}^{1}$. The zeros in the ferromagnetic case have subsequently become known as the Lee-Yang zeros. To study the zeros of $Z_{G}$ for all $G \in \mathcal{G}_{d+1}$ one can consider the subset of finite rooted Cayley trees with down-degree $d$, which we denote by $\mathcal{C}_{d+1}$. The Lee-Yang zeros of Cayley trees are studied in [MHZ75, MH77, BM97, BG01, CHJR19] among other papers. In all of these papers some variation of the following rational function plays an important role:

$$
f_{\lambda}(z)=f_{\lambda, d}(z)=\lambda \cdot\left(\frac{z+b}{b z+1}\right)^{d},
$$

where $f_{\lambda}$ is viewed as a function on the Riemann sphere. The significance of $f_{\lambda}$ in relation to the Cayley trees is explained by the following lemma.

Lemma 1.1. (E.g. [CHJR19, Proposition 1.1]) Let $b \in \mathbb{R}$ and $d \geq 2$. Then

$$
\mathcal{Z}_{\mathcal{C}_{d+1}}=\left\{\lambda: f_{\lambda}^{n}(\lambda)=-1 \text { for some } n \in \mathbb{Z}_{\geq 0}\right\} .
$$

Thus complex dynamical systems can be used to study the zeros of the partition function of Cayley trees. The following result from [PR20] shows that while the Cayley trees form a relatively small subset of the class of all graphs of bounded maximal degree, the zero free loci of these two classes are identical in the ferromagnetic case.

THEOREM 1.2. Let $d \geq 2$. If $0<b \leq(d-1) /(d+1)$ then

$$
\overline{\mathcal{Z}_{\mathcal{C}_{d+1}}}=\overline{\mathcal{Z}_{\mathcal{G}_{d+1}}}=\mathbb{S}^{1}
$$




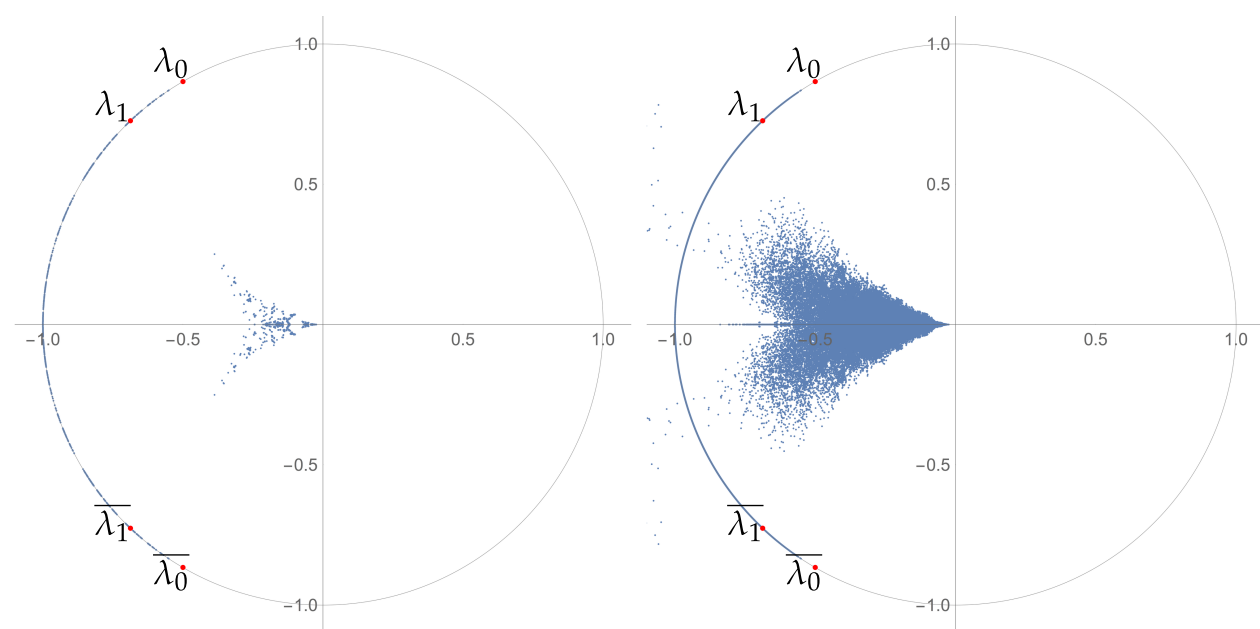

FIGURE 1. Comparing zeros of Cayley trees (left) and spherically symmetric trees (right) for $d=2$ and depth at most 11 .

If $(d-1) /(d+1)<b<1$ then

$$
\overline{\mathcal{Z}_{\mathcal{C}_{d+1}}}=\overline{\mathcal{Z}_{\mathcal{G}_{d+1}}}=\operatorname{Arc}\left[\lambda_{1}, \overline{\lambda_{1}}\right],
$$

where $\lambda_{1}=\lambda_{1}(b) \in \mathbb{S}^{1}$ is the unique parameter in the upper half plane for which $f_{\lambda}$ has a parabolic fixed point.

Given $\alpha, \beta$ on the unit circle, we will use the notation $\operatorname{Arc}[\alpha, \beta]$ for the closed circular arc from $\alpha$ to $\beta$, traveling counterclockwise, and similarly for open and half-open circular arcs.

When $b>1$ the Lee-Yang circle theorem fails, and the set of zeros of the partition function is considerably more complicated. Consider, for example, Figure 1, illustrating the location of zeros for Cayley trees and for the larger class of spherically symmetric trees, defined in Definition 1.7 below, both for maximal down-degree $d=2$ and maximal depth 11. The pictures are symmetric with respect to reflection in the unit circle, but only few zeros outside of the unit disk are depicted because of space limitations.

The pictures clearly demonstrate the appearance of zero parameters both on and off the unit circle. In this paper we focus on describing the set of zeros on the unit circle. Our main result shows that, contrary to the ferromagnetic case, the zero free locus for the Cayley trees is strictly larger than that of the class of all bounded degree graphs.

We recall the following result from [PR20].

THEOREM 1.3. Let $\lambda_{0}=e^{i \theta_{0}} \in \mathbb{S}^{1}$ be the parameter with the smallest positive angle $\theta_{0}$ for which $f_{\lambda_{0}}\left(\lambda_{0}\right)=1$. Then

$$
\mathcal{Z}_{\mathcal{G}_{d+1}} \cap \mathbb{R}_{+} \cdot \operatorname{Arc}\left[\overline{\lambda_{0}}, \lambda_{0}\right]=\emptyset,
$$

but

$$
\lambda_{0}, \overline{\lambda_{0}} \in \overline{\mathcal{Z}_{\mathcal{C}_{d+1}}}
$$


Note that, in Figure $1, \lambda_{0}$ and $\overline{\lambda_{0}}$ are depicted by the conjugate pair of points with smallest absolute argument. The other conjugate pair of points corresponds to $\lambda_{1}$ and $\overline{\lambda_{1}}$, having the same definition as in the ferromagnetic case.

Our main result is the following theorem.

THEOREM 1.4. Let $d \geq 2$. If $b \geq(d+1) /(d-1)$ then

$$
\overline{\mathcal{Z}_{\mathcal{C}_{d+1}} \cap \mathbb{S}^{1}}=\overline{\mathcal{Z}_{\mathcal{G}_{d+1}} \cap \mathbb{S}^{1}}=\mathbb{S}^{1} .
$$

If $1<b<(d+1) /(d-1)$ then:

(1) density for Cayley trees,

$$
\operatorname{Arc}\left[\lambda_{1}, \overline{\lambda_{1}}\right] \cup\left\{\lambda_{0}, \overline{\lambda_{0}}\right\} \subset \overline{\mathcal{Z}_{\mathcal{C}_{d+1}} \cap \mathbb{S}^{1}} ;
$$

(2) nowhere density for Cayley trees, the set

$$
\overline{\mathcal{Z}_{\mathcal{C}_{d+1}}} \cap \operatorname{Arc}\left[\lambda_{0}, \lambda_{1}\right]
$$

is a nowhere dense subset of $\operatorname{Arc}\left[\lambda_{0}, \lambda_{1}\right]$;

(3) density for arbitrary graphs, there exists $\lambda_{3} \in \operatorname{Arc}\left(\lambda_{0}, \lambda_{1}\right)$ such that

$$
\overline{\mathcal{Z}_{\mathcal{G}_{d+1}} \cap \operatorname{Arc}\left[\lambda_{0}, \lambda_{3}\right]}=\operatorname{Arc}\left[\lambda_{0}, \lambda_{3}\right] .
$$

Case (3) will be proved in $\S 6$, building upon results from earlier sections. Cases (1) and (2) will be proved respectively in $\S 4$ and 3 . The statement for $b \geq(d+1) /(d-1)$ is simpler and will be proved at the end of $\$ 3$.

Remark 1.5. The fact that the closure of $\mathcal{Z}_{\mathcal{C}_{d+1}}$ is strictly smaller than the closure of $\mathcal{Z}_{\mathcal{G}_{d+1}}$ also holds outside of the unit circle, a statement that is considerably easier to prove. For example, the solution to the one-dimensional Ising model gives the density of zeros in a real interval $\left[-\alpha^{-1},-\alpha\right]$, for some $\alpha \in(0,1)$. On the other hand, using Corollary 3.10, one can prove the existence of a neighbourhood of $\lambda=-1$ where all accumulation points of $\mathcal{Z}_{\mathcal{C}_{d+1}}$ must lie on the unit circle.

We prove case (3) for the subclass in $\mathcal{G}_{d+1}$ given by the spherically symmetric trees. These trees have the advantage that dynamical methods can be used to describe the location of zeros, as indicated by the following lemma, whose proof will be given later in this section.

LeMmA 1.6. Let $d \geq 2$ and $\lambda, b \in \mathbb{C}$. Then there exists a spherically symmetric tree $T$ with down-degree at most $d$ for which $Z_{T}(\lambda, b)=0$ if and only if

$$
g(\lambda)=-1
$$

for some $g \in H_{\lambda, d+1}$.

Here $H_{\lambda, d+1}=H_{\lambda, d+1}(b)$ is the rational semigroup generated by $f_{\lambda, 1}, \ldots, f_{\lambda, d}$, that is,

$$
H_{\lambda, d}=\left\langle f_{\lambda, 1}, \ldots, f_{\lambda, d}\right\rangle
$$


We will prove that a specific subsemigroup of $H_{\lambda, d}$ is hyperbolic for all $\lambda \in \operatorname{Arc}\left[\lambda_{0}, \lambda_{2}\right)$, for some $\lambda_{2} \in \operatorname{Arc}\left(\lambda_{0}, \lambda_{1}\right)$. Moreover, we obtain uniform bounds on the expansion rate on compact subsets of $\operatorname{Arc}\left[\lambda_{0}, \lambda_{2}\right)$. We also show that for any $\lambda \in \operatorname{Arc}\left[\lambda_{0}, \lambda_{1}\right]$, there exists a sequence in the subsemigroup for which +1 lies on the Julia set. Combining these two statements, we obtain uniform expansion along an orbit of +1 . The density of zero parameters is a consequence for $\lambda$ sufficiently close to $\lambda_{0}$.

We emphasize that in statement (3) of Theorem 1.4 we only consider zero parameters in $\mathbb{S}^{1}$. Alternatively we can consider $\overline{\mathcal{Z}_{\mathcal{G}_{d+1}}} \cap \mathbb{S}^{1}$, which is a priori a larger set. We prove in $\S 6$ that this closure contains the circular arc

$$
\operatorname{Arc}\left[\lambda_{0}, \lambda_{2}\right)
$$

which is the arc where the earlier discussed subsemigroup of $H_{\lambda, d}$ acts hyperbolically. The parameter $\lambda_{2}$ can be explicitly calculated. Computer evidence in fact suggests that

$$
\overline{\mathcal{Z}_{\mathcal{G}_{d+1}}} \cap \mathbb{S}^{1}=\operatorname{Arc}\left[\lambda_{0}, \overline{\lambda_{0}}\right] .
$$

In $\S 2$ we prove basic results regarding the attracting intervals of the maps $f_{\lambda}$, to be used in later sections. In $\$ 3$ we consider the hyperbolic components in the parameter space of the maps $f_{\lambda}$, and prove case (2) of Theorem 1.4. In $\S 4$ we consider only parameters $\lambda$ on the unit circle and prove case (1).

In the remainder of this introduction we recall the relationship between partition functions on Cayley trees and spherically symmetric trees on the one hand, and iteration and semigroup actions on the other hand. In particular, we give a short proof of Lemmas 1.1 and 1.6. In the rest of the paper we will only consider the two dynamical systems, with few references to partition functions.

1.1. Iterates and semigroups arising from trees. Let us recall from [PR20] (but see also [CHJR19]) how the zeros of the Ising partition function $Z_{G}(\lambda)$ on some recursively defined trees can be studied using iterations or compositions of rational functions.

Let $v$ be a marked node of a graph $G=(V, E)$. Note that

$$
Z_{G}=Z_{G, v}^{\text {in }}+Z_{G, v}^{\text {out }}
$$

where $Z_{G, v}^{\text {in }}$ sums only over $U \subset V$ with $v \in U$, and $Z_{G, v}^{\text {out }}$ sums only over $U \subset V$ with $v \notin U$. It follows that

$$
Z_{G}=0 \Leftrightarrow R_{G, v}:=\frac{Z_{G, v}^{\text {in }}}{Z_{G, v}^{\text {out }}}=-1 \text { or } Z_{G, v}^{\text {in }}=Z_{G, v}^{\text {out }}=0 .
$$

Suppose now that $G=T$ is a tree. Denote the neighbours of $v$ by $v_{1}, \ldots, v_{k}$, and the corresponding connected components of $G-v$ by $T_{1}, \ldots, T_{k}$. Then it follows that

$$
R_{T, v}=\lambda \prod_{i=1}^{k} \frac{R_{T_{i}, v_{i}}+b}{b R_{T_{i}, v_{i}}+1}
$$


Hence when all the rooted trees $\left(T_{i}, v_{i}\right)$ are isomorphic, one obtains

$$
R_{T, v}=\lambda\left(\frac{R_{T_{i}, v_{i}}+b}{b R_{T_{i}, v_{i}}+1}\right)^{k} .
$$

Definition 1.7. Let $d \geq 2$ and let $\omega=\left(k_{1}, k_{2}, \ldots\right) \in\{1, \ldots, d\}^{\mathbb{N}}$. Let $T_{0}$ be the rooted graph with a single vertex. Recursively define the trees $T_{1}, \ldots$ by letting $T_{n}$ consist of a root vertex $v$ of degree $k_{n}$, with each edge incident to $v$ connected to the root of a copy of $T_{n-1}$. We say that the rooted trees $T_{n}$ are spherically symmetric of degree at most $d$. Equivalently a rooted tree, with root $v$, is said to be spherically symmetric if all leaves have the same depth $n$, and all vertices of depth $1 \leq j<n$ have down-degree $k_{j}$. When all degrees $k_{n}$ are equal to $d$ the tree $T_{n}$ is said to be a (rooted) Cayley tree of degree $d$.

Note that for a spherically symmetric tree

$$
\begin{aligned}
Z_{n}^{\text {in }}(\lambda) & =\lambda\left(Z_{n-1}^{\text {in }}(\lambda)+b Z_{n-1}^{\text {out }}(\lambda)\right)^{k_{n}} \in \mathbb{R}[\lambda], \text { and } \\
Z_{n}^{\text {out }}(\lambda) & =\left(b Z_{n-1}^{\text {in }}(\lambda)+Z_{n-1}^{\text {out }}(\lambda)\right)^{k_{n}} \in \mathbb{R}[\lambda] .
\end{aligned}
$$

Since we will work with $b \notin\{-1,+1\}$ it follows by induction that $Z_{n}^{\text {in }}(\lambda)$ and $Z_{n}^{\text {in }}(\lambda)$ cannot both be equal to zero, from which it follows that

$$
Z_{G}=0 \Leftrightarrow R_{G, v}=-1 .
$$

Noting that $R_{T_{0}, v}=\lambda$, it follows for Cayley trees that

$$
R_{T_{n}, v}=f_{d}^{n}(\lambda)=f_{d}^{n+1}(+1)
$$

where

$$
f(z)=f_{d}(z)=\lambda\left(\frac{z+b}{b z+1}\right)^{d},
$$

while for spherically symmetric trees we obtain

$$
R_{T_{n}, v}=f_{\omega}^{n}(\lambda):=f_{k_{n}} \circ \cdots \circ f_{k_{1}}(\lambda) .
$$

Hence we have proved Lemmas 1.1 and 1.6.

Motivated by this discussion, we introduce the notation

$$
\mathcal{Z}_{f}:=\left\{\lambda \in \mathbb{C}: f^{n}(1)=-1 \text { for some } n \in \mathbb{N}\right\} .
$$

and

$$
\mathcal{Z}_{H}:=\left\{\lambda \in \mathbb{C}: f_{\omega}^{n}(1)=-1 \text { for some } n \in \mathbb{N}, \omega \in\{1, \ldots, d\}^{\mathbb{N}}\right\},
$$

where $H$ again refers to the semigroup $\left\langle f_{1}, \ldots, f_{d}\right\rangle$. Thus $\lambda \in \mathcal{Z}_{f}$ if and only if $Z_{G}(\lambda)=0$ for a Cayley tree $G$, while $\lambda \in \mathcal{Z}_{H}$ if and only if $Z_{G}(\lambda)=0$ for a spherically symmetric tree $G$.

\section{J-stable components}

Given a family of rational maps $f_{\lambda}$ parameterized by a complex manifold $\Lambda$, the set of $J$-stable parameters is the set of parameters for which the Julia set $J_{\lambda}$ moves continuously with respect to the Hausdorff topology. The concept of $J$-stability plays a central role in 
the study of rational functions. We refer the interested reader to [MnSS83, McM94, Slo91] for a more detailed description of $J$-stability.

Given a positive integer $d \geq 2$ and $b>1$, let $f_{\lambda}$ be the family of rational functions given by equation (1) parameterized by $\Lambda=\widehat{\mathbb{C}}$. We will write $\Lambda^{\text {stb }}$ for the set of $J$-stable parameters and $\Lambda^{\text {hyp }}$ for the set of hyperbolic parameters, that is, the values for which $f_{\lambda}$ has no critical points or parabolic cycles on $J_{\lambda}$. Recall that $\Lambda^{\text {stb }}$ is a dense open set and that the set $\Lambda^{\text {hyp }}$ is an open and closed subset of $\Lambda^{\text {stb }}$. Whether the equality $\Lambda^{\text {stb }}=\Lambda^{\text {hyp }}$ holds for the family given by equation (1) is a natural question, though not directly relevant for our purposes.

Given $\lambda \in \Lambda^{\text {stb }}$, we will write $\Lambda_{\lambda}^{\text {stb }}$ for the connected component of $\Lambda^{\text {stb }}$ containing the parameter $\lambda$.

THEOREM 2.1. There exists a holomorphic motion of $J_{\lambda}$ over $\left(\Lambda_{\lambda}^{\text {stb }}, \lambda\right)$ which respects the dynamics, that is, there exists a continuous map $\varphi: \widehat{\mathbb{C}} \times \Lambda_{\lambda}^{\text {stb }} \rightarrow \widehat{\mathbb{C}}$ satisfying the following properties.

(1) $\varphi_{\mu}$ is the identity at the base point $\lambda$, that is, $\varphi_{\lambda}(z)=z$.

(2) For every $z \in J_{\lambda}$ the map $\varphi_{\mu}(z)$ is holomorphic in $\mu \in \Lambda_{\lambda}^{\mathrm{stb}}$.

(3) For every $\mu \in \Lambda_{\lambda}^{\text {stb }}$ the map $z \mapsto \varphi_{\mu}(z)$ is injective and can be extended to $a$ quasi-conformal map $\varphi_{\mu}: \hat{\mathbb{C}} \rightarrow \hat{\mathbb{C}}$.

(4) For every $\mu \in \Lambda_{\lambda}^{\text {stb }}$ the map $\varphi_{\mu}: J_{\lambda} \rightarrow J_{\mu}$ is a homeomorphism. Furthermore, the following diagram commutes:

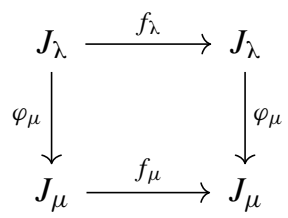

Such a map $\varphi$ satisfies the following two additional properties.

(5) Given $z \in \widehat{\mathbb{C}}$, the map $\varphi_{\mu}^{-1}(z)$ is continuous with respect to $\mu$,

(6) Let $z_{n} \rightarrow z$ be a convergent sequence and assume that $\mu \mapsto \varphi_{\mu}(z)$ is not constant.

Then there exist a subsequence $n_{k}$ and $\mu_{k} \rightarrow \lambda$ so that

$$
\varphi_{\mu_{k}}\left(z_{n_{k}}\right)=z
$$

Remark 2.2. The existence of a continuous map $\varphi$ satisfying properties (1)-(4) was proven by [MnSS83, Slo91], while the properties (5) and (6) follow immediately from continuity of $\varphi$. The holomorphic motion is unique on the Julia set $J_{\lambda}$, in the sense that any other continuous map $\tilde{\varphi}$ which satisfies properties (1)-(4) has to agree with $\varphi$ on the set $J_{\lambda} \times \Lambda_{\lambda}^{\mathrm{stb}}$.

Define the two sets

$$
\mathcal{F}:=\left\{\lambda \in \widehat{\mathbb{C}} \mid 1 \in F_{\lambda}\right\}, \quad \mathcal{J}:=\left\{\lambda \in \widehat{\mathbb{C}} \mid 1 \in J_{\lambda}\right\} .
$$

Given a connected component $U \subset \Lambda^{\text {stb }}$, we further write $\mathcal{F}_{U}=\mathcal{F} \cap U$ and $\mathcal{J}_{U}=\mathcal{J} \cap U$. Since the Julia set $J_{\lambda}$ moves continuously for $\lambda \in U$, it follows that $\mathcal{F}_{U}$ is open while $\mathcal{J}_{U}$ is closed with respect the intrinsic topology of $U$. 
Definition 2.3. Let $U$ be a connected component of $\Lambda^{\text {stb }}$. We say that $U$ is exceptional if there exists $\lambda \in \mathcal{J}_{U}$ so that $\mu \mapsto \varphi_{\mu}(1)$ is constant, where $\varphi_{\mu}$ denotes the holomorphic motion of $J_{\lambda}$ over $(U, \lambda)$.

Remark 2.4. Suppose that $U$ is an exceptional component of $\Lambda^{\text {stb }}$ and let $\lambda \in U$ be so that the map $\mu \mapsto \varphi_{\mu}(1)$ is constant. Given another $\tilde{\lambda} \in U$, we have that $\varphi_{\tilde{\lambda}}(1)=\varphi_{\lambda}(1)=1$, and therefore that $1 \in J_{\tilde{\lambda}}$. Let $\widetilde{\varphi}_{\mu}$ be the holomorphic motion of $J_{\tilde{\lambda}}$ over $(U, \tilde{\lambda})$. Then we have

$$
\widetilde{\varphi}_{\mu}(1)=\varphi_{\mu} \circ \varphi_{\widetilde{\lambda}}^{-1}(1)=1 \text { for all } \mu \in U .
$$

This shows that if $U$ is an exceptional component then $\mathcal{J}_{U}=U$ and for every $\lambda \in U$ the map $\mu \mapsto \varphi_{\mu}(1)$ is constant.

Proposition 2.5. Let $U$ be a connected component of $\Lambda^{\mathrm{stb}}$. Then the set $\mathcal{J}_{U}$ is perfect with respect to the intrinsic topology of $U$.

Proof. We already know that $\mathcal{J}_{U}$ is closed in $U$, thus we only have to show that $\mathcal{J}_{U}$ contains no isolated points. If $U$ is an exceptional hyperbolic component, then according to Remark 2.4 we have $\mathcal{J}_{U}=U$ and the result follows immediately. Assume instead that $U$ is not exceptional, let $\lambda \in \mathcal{J}_{U}$ and $\varphi_{\mu}$ be the holomorphic motion of $J_{\lambda}$ over $(U, \lambda)$.

Since the Julia set of a rational map is perfect, it follows that we may take $z_{n} \in J_{\lambda}$ which converges to 1 , and is not identically equal to 1 . By Theorem 2.1 we may therefore find a sequence $n_{k} \geq 0$ and $\mu_{k} \rightarrow \lambda$ so that $\varphi_{\mu_{k}}\left(z_{n_{k}}\right)=1$ for every $k$. Since $\varphi_{\mu}\left(J_{\lambda}\right)=J_{\mu}$ we conclude that $\mu_{k} \in \mathcal{J}_{U}$, proving that $\lambda$ is not an isolated point of $\mathcal{J}_{U}$, and that $\mathcal{J}_{U}$ is perfect.

The definition of active parameters is classical [McM00, DF08], and was inspired by [Lev81, Lyu83]. In all these works activity is always defined in terms of the family $\left\{f_{\lambda}^{n}\right.$ 。 $c(\lambda)$ \}, where $c(\lambda)$ is the parameterization of a critical point. For our purpose it is natural to replace $c(\lambda)$ with the point 1 , even though the point 1 is never critical.

Definition 2.6. A parameter $\lambda \in \widehat{\mathbb{C}}$ is passive if the family $\left\{\lambda \mapsto f_{\lambda}^{n}(1)\right\}_{n \in \mathbb{N}}$ is normal in some neighbourhood of $\lambda$, and is active otherwise.

We further remark that, given a marked point $a(\lambda)$ and the corresponding family $\left\{f_{\lambda}^{n} \circ\right.$ $a(\lambda)\}$, it would be more accurate to say that the marked point $a(\lambda)$ is passive/active at $\lambda$. However, since in our case the marked point is always 1 , we will refer to passive/active parameters instead.

LEMMA 2.7. Every active parameter is in $\overline{\mathcal{Z}_{\mathcal{C}_{d+1}}}$.

Proof. This is a standard normality argument. Assume first that $d \neq 2$ or that $\lambda \neq-1$. Let $\lambda$ be an active parameter and choose $\alpha_{0}, \beta_{0} \in f_{\lambda}^{-1}(\{-1\})$ so that $\left\{-1, \alpha_{0}, \beta_{0}\right\}$ are all distinct. Since -1 is never a critical value of $f_{\lambda}$ we can define two holomorphic maps $\alpha_{\mu}, \beta_{\mu}$ so that $f_{\mu}\left(\left\{\alpha_{\mu}, \beta_{\mu}\right\}\right)=-1$ in a neighbourhood of $\lambda$. By conjugating with a holomorphically varying family of Möbius transformation we may assume that $\left\{-1, \alpha_{\mu}, \beta_{\mu}\right\}=\{-1,0, \infty\}$. Since the family $\left\{f_{\mu}^{n}(1)\right\}$ is not normal at $\lambda$, by Montel's 
theorem we conclude that it cannot avoid the three points $\{-1,0, \infty\}$ in a neighbourhood of $\lambda$. Since $\{0, \infty\}$ are both mapped to -1 , the orbit $f_{\lambda}^{n}(1)$ cannot miss the point -1 near $\lambda$, proving that $\lambda \in \overline{\mathcal{Z}_{\mathcal{C}_{d+1}}}$. When $d=2$ and $\lambda=-1$ the point -1 is fixed and has only two preimages $\{1,-1\}$. In this case we fix $\alpha_{0}=1$ and choose $\gamma_{0}$ as one of the two preimages of 1 ; the proof is then the same as above.

Lemma 2.8. Let $U$ be a non-exceptional component of $\Lambda^{\text {stb }}$. Then every $\lambda \in \mathcal{F}_{U}$ is passive and every $\lambda \in \mathcal{J}_{U}$ is active.

Proof. Given $\lambda \in U$, let $\varphi_{\mu}$ be the holomorphic motion of $J_{\lambda}$ over $(U, \lambda)$. If $\lambda \in \mathcal{F}_{U}$ then the orbit $f_{\lambda}^{n}(1)$ avoids the Julia set $J_{\lambda}$ and, in particular, it avoids three distinct points $\{a, b, c\} \subset J_{\lambda}$. Since the set $\mathcal{F}_{U}$ is open we have that $1 \in F_{\mu}$ for every $\mu$ sufficiently close to $\lambda$, and therefore the orbit of $f_{\mu}^{n}(1)$ avoids the set $\varphi_{\mu}(\{a, b, c\}) \subset J_{\mu}$. Using the normality argument from the proof of the previous lemma, we may therefore conclude that $\left\{f_{\mu}^{n}(1)\right\}$ is normal in a neighbourhood of $\lambda$, showing that $\lambda$ is passive.

Suppose that there exists $\lambda \in \mathcal{J}_{U}$ which is passive. Given any $0<\varepsilon<\operatorname{diam}\left(J_{\lambda}\right) / 2$, by equicontinuity we can find $\delta>0$ so that

$$
\left|f_{\mu}^{n}(1)-f_{\lambda}^{n}(1)\right|<\varepsilon / 2 \text { for all } \mu \in B(\lambda, \delta) \text { and } n \in \mathbb{N} .
$$

Given any open neighbourhood $U \ni 1$, there exists $N \in \mathbb{N}$ so that $f_{\lambda}^{N}\left(U \cap J_{\lambda}\right)=J_{\lambda}$. Given $w \in J_{\lambda}$ with distance at least $\varepsilon$ from $f_{\lambda}^{N}(1)$, we can therefore find $z \in U \cap J_{\lambda}$ so that $f_{\lambda}^{N}(z)=w$. We conclude that we can construct a sequence $z_{k} \in J_{\lambda}$ converging to the point 1 and a sequence of positive integers $N_{k}$ so that

$$
\left|f_{\lambda}^{N_{k}}\left(z_{k}\right)-f_{\lambda}^{N_{k}}(1)\right|>\varepsilon
$$

By Theorem 2.1, up to taking a subsequence of $z_{k}$ if necessary, we may assume that there exists a sequence $\mu_{k} \in B(\lambda, \delta)$ so that $\mu_{k} \rightarrow \lambda$ and so that $\varphi_{\mu_{k}}\left(z_{k}\right)=1$. This implies that

$$
\left|\varphi_{\mu_{k}} \circ f_{\lambda}^{N_{k}}\left(z_{k}\right)-f_{\lambda}^{N_{k}}(1)\right|=\left|f_{\mu_{k}}^{N_{k}}(1)-f_{\lambda}^{N_{k}}(1)\right|<\varepsilon / 2 .
$$

By continuity of the holomorphic motion, we may further assume that whenever $k$ is sufficiently large we have

$$
\left|\varphi_{\mu_{k}} \circ f_{\lambda}^{N_{k}}\left(z_{k}\right)-f_{\lambda}^{N_{k}}\left(z_{k}\right)\right|=\left|\varphi_{\mu_{k}} \circ f_{\lambda}^{N_{k}}\left(z_{k}\right)-\varphi_{\lambda} \circ f_{\lambda}^{N_{k}}\left(z_{k}\right)\right|<\varepsilon / 2 .
$$

In combination with the previous inequality we conclude that for every $k$ sufficiently large we have

$$
\left|f_{\lambda}^{N_{k}}\left(z_{k}\right)-f_{\lambda}^{N_{k}}(1)\right|<\varepsilon \quad \text { for all } n \in \mathbb{N},
$$

contradicting the definition of the sequence $z_{k}$. Thus every $\lambda \in \mathcal{J}_{U}$ is active.

3. Dynamics of the map $f_{\lambda}$

For given $d \geq 2$ and $b>1$, we are interested in the dynamics of the map $f_{\lambda}$ under the assumption that $\lambda \in \mathbb{S}^{1}$. In this case we have

$$
\begin{aligned}
& f_{\lambda}: B(0,1) \rightarrow \widehat{\mathbb{C}} \backslash \overline{B(0,1)} \rightarrow B(0,1), \\
& f_{\lambda}: \mathbb{S}^{1} \rightarrow \mathbb{S}^{1},
\end{aligned}
$$


and the restriction of $f$ to $\mathbb{S}^{1}$ is orientation reversing. If we write respectively $F_{\lambda}$ and $J_{\lambda}$ for the Fatou and Julia set of the map $f_{\lambda}$, we conclude that

$$
F_{\lambda} \supset \hat{\mathbb{C}} \backslash \mathbb{S}^{1}, \quad J_{\lambda} \subset \mathbb{S}^{1} \text { for all } \lambda \in \mathbb{S}^{1}
$$

When $\lambda \in \mathbb{S}^{1}$, we further have

$$
\left|f_{\lambda}^{\prime}(z)\right|=\frac{d\left(b^{2}-1\right)}{1+b^{2}+2 b \operatorname{Re} z} \quad \text { for all } z \in \mathbb{S}^{1} .
$$

Therefore the value of $\left|f_{\lambda}^{\prime}(z)\right|$ increases as Re $z$ decreases. Recall that a rational map $f$ is expanding on an invariant set $K$ if $f$ locally increases distances, while it is uniformly expanding if distances are locally increased by a multiplicative factor, bounded below by a constant strictly greater than 1 .

LEMMA 3.1. [PR20, Lemma 9] If $b>(d+1) /(d-1)$ and $\lambda \in \mathbb{S}^{1}$ then the map $f_{\lambda}$ is uniformly expanding on $\mathbb{S}^{1}$. If $b=(d+1) /(d-1)$ then the map $f_{\lambda}$ is expanding on $\mathbb{S}^{1}$.

Definition 3.2. [PR20, Lemma 12] Given $1<b<(d+1) /(d-1)$, we write $\lambda_{1} \in \mathbb{S}^{1}$ for the unique parameter satisfying $0<\arg \left(\lambda_{1}\right)<\pi$ and for which $f_{\lambda_{1}}$ has a parabolic fixed point.

The following proposition describes the set of hyperbolic parameters on the unit circle.

Proposition 3.3. We have

$$
\mathbb{S}^{1} \cap \Lambda^{\text {hyp }}= \begin{cases}\mathbb{S}^{1} & \text { if } b>\frac{d+1}{d-1}, \\ \mathbb{S}^{1} \backslash\{1\} & \text { if } b=\frac{d+1}{d-1}, \\ \mathbb{S}^{1} \backslash\left\{\lambda_{1}, \bar{\lambda}_{1}\right\} & \text { if } 1<b<\frac{d+1}{d-1} .\end{cases}
$$

Proof. When $b>(d+1) /(d-1)$, by Lemma 3.1 the map $f_{\lambda}$ is uniformly expanding and therefore hyperbolic. When $b=(d+1) /(d-1)$ and $\lambda \in \mathbb{S}^{1} \backslash\{1\}$, for every $z \in \mathbb{S}^{1}$ either $z$ or $f(z)$ is uniformly bounded away from 1 . By (4) we obtain again that the map $f_{\lambda}^{2}$ is uniformly expanding, proving that $f_{\lambda}$ is hyperbolic. On the other hand when $\lambda=1$ the map $f_{\lambda}$ has a parabolic fixed point, and therefore it is not hyperbolic.

Given $1<b<(d+1) /(d-1), \lambda_{1}, \bar{\lambda}_{1}$ are the unique parameters on the unit circle for which $f_{\lambda}$ has a parabolic fixed point. Suppose that there exists $\lambda \in \mathbb{S}^{1} \backslash\left\{\lambda_{1}, \bar{\lambda}_{1}\right\}$ which is not hyperbolic. By (3) the set $\widehat{\mathbb{C}} \backslash \mathbb{S}^{1}$ is contained in the Fatou set, and therefore the critical points of $f_{\lambda}$ are also contained in the Fatou set. It follows that the map $f_{\lambda}$ must have a parabolic cycle with period at least 2. Since there are at most two Fatou components we conclude that the period of the parabolic cycle is exactly 2 .

Notice that for every $\lambda \in \mathbb{S}^{1}$ we have that $f_{\lambda}(1 / \bar{z})=1 / \overline{f_{\lambda}(z)}$, and therefore that

$$
f_{\lambda}^{n}(-1 / b)=1 / \overline{f_{\lambda}^{n}(-b)}
$$


Let $z_{1}, z_{2} \in \mathbb{S}^{1}$ be the parabolic cycle of $f_{\lambda}$. These two points are parabolic fixed points for $f_{\lambda}^{2}$ and both of them have an immediate basin that must coincide with a Fatou component of $f_{\lambda}$. By replacing $z_{1}$ with $z_{2}$ if necessary, we may therefore assume that $B(0,1)$ is the attracting basin of $z_{1}$, while $\widehat{\mathbb{C}} \backslash \bar{B}(0,1)$ is the attracting fixed point of $z_{2}$. This shows that $f_{\lambda}^{2 n}(-1 / b) \rightarrow z_{1}$ and that $f_{\lambda}^{2 n}(-b) \rightarrow z_{2}$, and therefore that $z_{1}=1 / \bar{z}_{2}=z_{2}$, contradicting the fact that the period of the cycle is 2 .

We notice that -1 is always a hyperbolic parameter, therefore the set $\Lambda_{-1}^{\text {hyp }}$, that is, the connected component of $\Lambda^{\text {hyp }}$ containing -1 , is always well defined. On the other hand, the set $\Lambda_{1}^{\text {hyp }}$ is defined for $b \neq(d+1) /(d-1)$ and does not coincide with $\Lambda_{-1}^{\text {hyp }}$ if and only if $1<b<(d+1) /(d-1)$.

Proposition 3.4. Let $b>1$. Then for every $\lambda \in \Lambda_{-1}^{\text {hyp }}$ the Julia set $J_{\lambda}$ is a quasi-circle, while the Fatou set $F_{\lambda}$ contains exactly two components which are the attracting basin of a (super)attracting 2-cycle. If we further assume that $|\lambda|=1$ then $J_{\lambda}=\mathbb{S}^{1}$.

Let $1<b<(d+1) /(d-1)$. Then for every $\lambda \in \Lambda_{1}^{\text {hyp }}$ the Julia set $J_{\lambda}$ is a Cantor set, while the Fatou set $F_{\lambda}$ coincides with the attracting component of an attracting fixed point.

Proof. The function $g(z)=f_{-1}^{2}(z)-z$ satisfies $g(0)<0$ and $g(-1 / b)>0$. Since $g$ is a real map and $f_{-1}$ maps the disk to the complement of its closure, we conclude that $f_{-1}$ has a periodic point of order 2 in $B(0,1)$. By (3) it is clear that $J_{-1}=\mathbb{S}^{1}$. The holomorphic motion of $J_{-1}$ over $\left(\Lambda_{-1}^{\text {hyp }},-1\right)$ given by Theorem 2.1 now implies that the Julia set $J_{\lambda}$ is a quasi-circle for every $\lambda \in \Lambda_{-1}^{\text {hyp }}$. The two components of $F_{-1}$ are mapped into each other, and by continuity the same holds for $F_{\lambda}$. Hyperbolicity of $f_{\lambda}$ implies that they are the basin of a (super)attracting 2-cycle.

When $1<b<(d+1) /(d-1)$ the map $f_{1}$ has an attracting fixed point at 1 . It is well known that the Julia set of a rational function with a single invariant attracting basin containing all the critical points is a Cantor set (see also [Mil00, Theorem B.1]). Proceeding as above, we obtain that for every $\lambda \in \Lambda_{1}^{\text {hyp }}$ the set $J_{\lambda}$ is a Cantor set and that $F_{\lambda}$ coincides with the attracting basin of a (super)attracting fixed point. Since the critical points of $f_{\lambda}$ are not fixed points, we conclude that the fixed point is attracting.

Remark 3.5. A bicritical rational map is a rational map with two distinct critical points (counted without multiplicity). The space of bicritical rational maps of degree $d$ was studied by Milnor [Mil00], where he shows that its Moduli space (the space of holomorphic conjugacy classes) is biholomorphic to $\mathbb{C}^{2}$. In that paper he constructs explicit conjugacy invariants $f \mapsto(X, Y)$. In our case the invariants associated to the map $f_{\lambda}$ are given by

$$
X=\frac{b^{2}}{1-b^{2}}, \quad Y=\left(\lambda+\frac{1}{\lambda}\right) \frac{b^{d-1}}{\left(1-b^{2}\right)^{d}} .
$$

A bicritical rational map is real if its invariants are real, or equivalently if there exists an antiholomorphic involution $\alpha$ which commutes with the map. When $b \in \mathbb{R} \backslash\{0,1\}$, the map $f_{\lambda}$ is real if and only if $\lambda \in \mathbb{S}^{1}$, and the corresponding involution is $\alpha=1 / \bar{z}$. The 

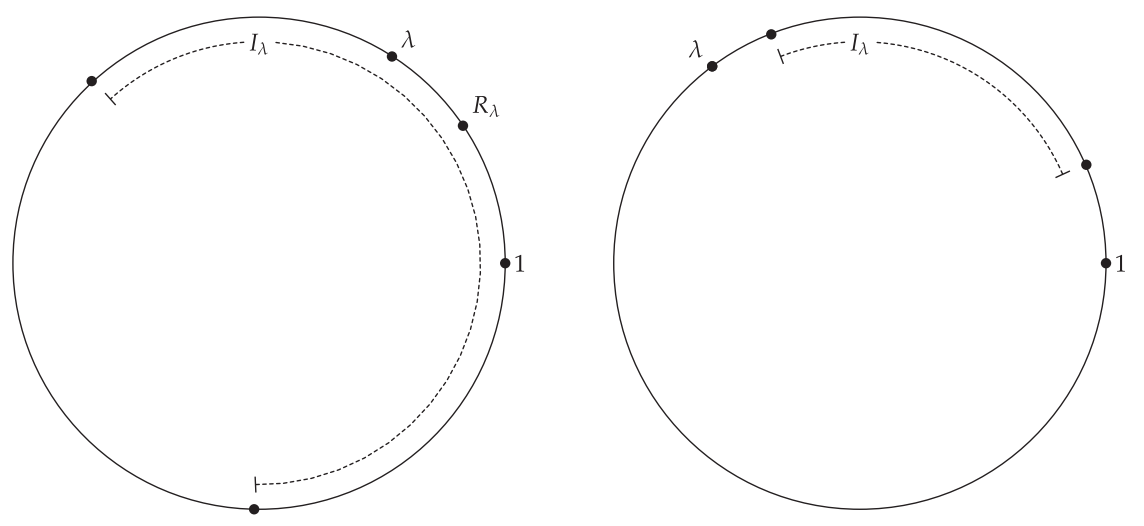

FIgURE 2. The position of $I_{\lambda}$ for $\lambda \in \operatorname{Arc}\left(1, \lambda_{0}\right)$ and for $\lambda \in \operatorname{Arc}\left(\lambda_{0}, \lambda_{1}\right)$.

results obtained by Milnor for real maps are sufficient to conclude that, given $\lambda \in \mathbb{S}^{1}$, the Julia set is either a Cantor set or the whole circle.

The following definition follows from the proposition above. Recall that when $1<$ $b<(d+1) /(d-1)$ by Proposition 3.3 we have $\operatorname{Arc}\left(\overline{\lambda_{1}}, \lambda_{1}\right)=\Lambda_{1}^{\text {hyp }} \cap \mathbb{S}^{1}$ and that when $\lambda \in \mathbb{S}^{1}$ all fixed points of $f_{\lambda}$ are on the unit circle.

Definition 3.6. Given $1<b<(d+1) /(d-1)$ and $\lambda \in \operatorname{Arc}\left(\overline{\lambda_{1}}, \lambda_{1}\right)$, we write $R_{\lambda} \in \mathbb{S}^{1}$ for the attracting fixed point of $f_{\lambda}$ and $I_{\lambda}$ for the connected component of $F_{\lambda} \cap \mathbb{S}^{1}$ containing $R_{\lambda}$. Notice that the map $f_{\lambda}$ is an orientation-reversing bijection $f_{\lambda}: I_{\lambda} \rightarrow I_{\lambda}$.

THEOREM 3.7. Let $1<b<(d+1) /(d-1)$. Then there exist unique parameters $\lambda_{0} \in \mathbb{S}^{1}$ with $0<\arg \left(\lambda_{0}\right)<\arg \left(\lambda_{1}\right)<\pi$, so that when $\lambda \in \operatorname{Arc}\left[1, \lambda_{1}\right)$,

$$
\begin{cases}\operatorname{Arc}(1, \lambda) \Subset I_{\lambda} & \text { for } \lambda \in \operatorname{Arc}\left[1, \lambda_{0}\right), \\ I_{\lambda}=\operatorname{Arc}(1, \lambda) & \text { for } \lambda=\lambda_{0}, \\ I_{\lambda} \Subset \operatorname{Arc}(1, \lambda) & \text { for } \lambda \in \operatorname{Arc}\left(\lambda_{0}, \lambda_{1}\right) .\end{cases}
$$

Similar inclusions hold for $\lambda \in\left(\bar{\lambda}_{1}, 1\right]$.

The interval $I_{\lambda}$ is illustrated in Figure 2 for the first and third cases. The existence of $\lambda_{0}$ and the first two inclusions follows from [PR20, Theorem 5]. Since the dynamics of $f_{\lambda}$ is conjugate to the dynamics of $f_{\bar{\lambda}}$ it will be sufficient to prove that $I_{\lambda} \Subset \operatorname{Arc}(1, \lambda)$ for $\lambda \in \operatorname{Arc}\left(\lambda_{0}, \lambda_{1}\right)$.

By the implicit function theorem the point $R_{\lambda}$ moves holomorphically in a neighbourhood of $\operatorname{Arc}\left(\overline{\lambda_{1}}, \lambda_{1}\right)$. Furthermore, by (4) it satisfies

$$
\operatorname{Re} R_{\lambda}>\frac{b^{2}(d-1)-(d+1)}{2 b}>-1 .
$$

Lemma 3.8. Let $1<b<(d+1) /(d-1)$. Then for every $\lambda \in \operatorname{Arc}\left(1, \lambda_{1}\right)$ we have $\operatorname{Im} R_{\lambda}>0$. 
Proof. A simple calculation shows that 1 is an attracting fixed point for $f_{\lambda}$ if and only if $\lambda=1$. This fact, together with (5), implies that $R_{1}=1$ and that $R_{\lambda} \neq \pm 1$ as $\lambda \in \operatorname{Arc}\left(1, \lambda_{1}\right)$. If we differentiate both sides of the equation $R_{\lambda}=f_{\lambda}\left(R_{\lambda}\right)$ with respect to $\lambda$ and evaluate at $\lambda=1$, we obtain

$$
\left.\partial_{\lambda} R_{\lambda}\right|_{\lambda=1}=\left.\frac{R_{\lambda}}{\lambda\left(1-f_{\lambda}^{\prime}\left(R_{\lambda}\right)\right)}\right|_{\lambda=1}=\frac{1}{1-d(1-b) /(1+b)}>0 .
$$

Therefore for $\lambda \in \operatorname{Arc}\left(1, \lambda_{1}\right)$ sufficiently close to 1 the point $R_{\lambda}$ lies in the upper half plane. However as $\lambda$ varies within $\operatorname{Arc}\left(1, \lambda_{1}\right)$, the point $R_{\lambda}$ moves on $\mathbb{S}^{1}$ without intersecting $\{-1,1\}$. Therefore $\operatorname{Im} R_{\lambda}>0$ on the whole of $\operatorname{Arc}\left(1, \lambda_{1}\right)$.

Proof of Theorem 3.7. Let $z_{\lambda}, w_{\lambda} \in \mathbb{S}^{1}$ so that $I_{\lambda}=\operatorname{Arc}\left(z_{\lambda}, w_{\lambda}\right)$. Since the map $f_{\lambda}$ : $I_{\lambda} \rightarrow I_{\lambda}$ is an orientation-reversing bijection, we have

$$
f_{\lambda}\left(z_{\lambda}\right)=w_{\lambda}, \quad f_{\lambda}\left(w_{\lambda}\right)=z_{\lambda},
$$

showing that $z_{\lambda}, w_{\lambda}$ are fixed points for $f_{\lambda}^{2}$. The Fatou set is connected, therefore there can be only one attracting or parabolic fixed point for $f_{\lambda}^{2}$, which is $R_{\lambda}$. This shows that the cycle $z_{\lambda}, w_{\lambda}$ is repelling. By the implicit function theorem the points $z_{\lambda}, w_{\lambda}$ move holomorphically and without collisions on some neighbourhood $U \supset \operatorname{Arc}\left(\lambda_{0}, \lambda_{1}\right)$.

By the previous lemma and (5) we have

$$
R_{\lambda} \in\left\{x+i y \mid y>0, x>\frac{b^{2}(d-1)-(d+1)}{2 b}>-1\right\} .
$$

Suppose now that for some $\lambda \in \operatorname{Arc}\left(\lambda_{0}, \lambda_{1}\right)$ we have $z_{\lambda} \in \operatorname{Arc}\left(1, R_{\lambda}\right)$. By (4) the map $f_{\lambda}$ is a contraction on $\operatorname{Arc}\left[1, R_{\lambda}\right]$. As the point $z$ moves counterclockwise on $\operatorname{Arc}\left[1, R_{\lambda}\right]$, its image $f_{\lambda}(z)$ moves clockwise on $\mathbb{S}^{1}$, starting at $\lambda$ and ending at $R_{\lambda}$. Since $f_{\lambda}$ is a contraction and $\operatorname{Im} R_{\lambda}>0$, this is possible only if $f_{\lambda}: \operatorname{Arc}\left[1, R_{\lambda}\right] \rightarrow \operatorname{Arc}\left[R_{\lambda}, \lambda\right]$ is an orientation-reversing bijection. We conclude that $w_{\lambda}=f_{\lambda}\left(z_{\lambda}\right) \in \operatorname{Arc}\left(R_{\lambda}, \lambda\right)$ and thus that $I_{\lambda} \Subset \operatorname{Arc}(1, \lambda)$.

If we differentiate both sides of $z_{\lambda}=f_{\lambda}^{2}\left(z_{\lambda}\right)$ we obtain the equation

$$
\partial_{\lambda} z_{\lambda}\left(1-\left(f_{\lambda}^{2}\right)^{\prime}\left(z_{\lambda}\right)\right)=\lambda^{-1}\left(z_{\lambda}+f_{\lambda}^{\prime}\left(w_{\lambda}\right) w_{\lambda}\right) .
$$

Since $I_{\lambda_{0}}=\operatorname{Arc}(1, \lambda)$, it follows that $z_{\lambda_{0}}=1$ is a repelling fixed point for $f_{\lambda_{0}}^{2}$. Furthermore, since $\left|f_{\lambda_{0}}^{\prime}(1)\right|<1$ we must have $\left|f_{\lambda_{0}}^{\prime}\left(\lambda_{0}\right)\right|>1$ and $\left|\left(f_{\lambda_{0}}^{2}\right)^{\prime}(1)\right|>1$. If we evaluate the expression above at $\lambda=\lambda_{0}$ we obtain that

$$
\left.\partial_{\lambda} z_{\lambda}\right|_{\lambda=\lambda_{0}}=\frac{1}{\lambda_{0}} \frac{1-\left|f_{\lambda_{0}}^{\prime}\left(\lambda_{0}\right)\right|}{1-\left|\left(f_{\lambda_{0}}^{2}\right)^{\prime}(1)\right|}=\frac{C}{\lambda_{0}},
$$

for some positive constant $C>0$. If we write $\lambda(\varepsilon)=\lambda_{0} e^{i \varepsilon}$ then we obtain that

$$
z_{\lambda(\varepsilon)}=1+i C \varepsilon+O\left(\varepsilon^{2}\right)
$$

therefore as $\lambda \in \operatorname{Arc}\left(\lambda_{0}, \lambda_{1}\right)$ is sufficiently close to $\lambda_{0}$, we must have $z_{\lambda} \in \operatorname{Arc}\left(1, R_{\lambda}\right)$ and thus that $I_{\lambda} \Subset \operatorname{Arc}(1, \lambda)$. 
This also proves that the point $z_{\lambda}$ moves counterclockwise as $\lambda$ is close to $\lambda_{0}$. We will show that $z_{\lambda}$ moves counterclockwise on the whole arc between $\lambda_{0}$ and $\lambda_{1}$. Assume otherwise. Then there is some $\mu \in \operatorname{Arc}\left(\lambda_{0}, \lambda_{1}\right)$ such that

$$
0=\left.\partial_{\lambda} z_{\lambda}\right|_{\lambda=\mu}=\frac{1}{\mu}\left(z_{\mu}+f_{\mu}^{\prime}\left(w_{\mu}\right) w_{\mu}\right) .
$$

Note that, since $z_{\lambda} \neq R_{\lambda}$ for any $\lambda \in \operatorname{Arc}\left(\lambda_{0}, \lambda_{1}\right)$, it follows that $\mu \in \operatorname{Arc}\left(1, R_{\mu}\right)$. As a result we must have $\left|f_{\mu}\left(z_{\mu}\right)\right|<1$, and therefore

$$
\left|f_{\mu}^{\prime}\left(z_{\mu}\right)\right| \cdot\left|f_{\mu}^{\prime}\left(w_{\mu}\right)\right|=\left|f_{\mu}^{\prime}\left(z_{\mu}\right)\right| \cdot\left|\frac{z_{\mu}}{w_{\mu}}\right|<1,
$$

which contradicts the fact that $z_{\mu}$ is a repelling fixed point of $f_{\mu}^{2}$. This shows that $z_{\lambda} \in$ $\operatorname{Arc}\left(1, R_{\lambda}\right)$ for every $\lambda \in \operatorname{Arc}\left(\lambda_{0}, \lambda_{1}\right)$ and therefore $I_{\lambda} \Subset \operatorname{Arc}(1, \lambda)$, concluding the proof of the proposition.

Recall that for $b>1$ the point -1 is a hyperbolic parameter and that $\Lambda^{\text {hyp }}$ is an open and closed subset of $\Lambda^{\text {stb }}$. Therefore the connected component $\Lambda_{-1}^{\text {hyp }}$ is a connected component of $\Lambda^{\mathrm{stb}}$. The same is clearly true for $\Lambda_{1}^{\text {hyp }}$ when 1 is a hyperbolic parameter.

LEMMA 3.9. For $b>1$ the component $\Lambda_{-1}^{\text {hyp }}$ is not exceptional. For $1<b<$ $(d+1) /(d-1)$ the component $\Lambda_{1}^{\text {hyp }}$ is not exceptional.

Proof. When $1<b<(d+1) /(d-1)$ the map $f_{\lambda}$ has an attracting fixed point at $z=1$ for $\lambda=1$, but not for nearby values of $\lambda$. The attracting fixed point therefore varies holomorphically and non-constantly as $\lambda$ varies, from which it follows that $\varphi_{\lambda}(1)$ is non-constant for $\lambda$ near 1 . Hence the component $\Lambda_{1}^{\text {hyp }}$ is not exceptional.

Suppose now that $\Lambda_{-1}^{\text {hyp }}$ is exceptional for some $b>1$ and let $\varphi_{\mu}$ be the holomorphic motion of $J_{-1}$ over $\left(\Lambda_{-1}^{\text {hyp }},-1\right)$. Since the holomorphic motion respects the dynamics we obtain that for every $\mu \in \Lambda_{-1}^{\text {hyp }}$,

$$
f_{\mu}^{n}(1)=f_{\mu}^{n} \circ \varphi_{\mu}(1)=\varphi_{\mu} \circ f_{-1}^{n}(1) \text {. }
$$

This shows that when the degree $d$ is even the function $f_{\mu}$ maps 1 to a fixed point of $f_{\mu}$, proving that $f_{\mu}^{2}(1)=f_{\mu}(1)$. On the other hand, when $d$ is odd the point 1 is periodic with period 2 , and therefore $f_{\mu}^{2}(1)=1$. Once the values of $b$ and $d$ are fixed there are only finitely many values of $\mu \in \Lambda_{-1}^{\text {hyp }}$ for which equation (7) is satisfied, giving a contradiction.

COROLlary 3.10. Suppose that $b>1$ and $U=\Lambda_{-1}^{\text {hyp }}$, or alternatively that $1<b<$ $(d+1) /(d-1)$ and $U=\Lambda_{1}^{\text {hyp }}$. Then the set of accumulation points of $\mathcal{Z}_{\mathcal{C}_{d+1}}$ in $U$ equals $\mathcal{J}_{U}$. Moreover, if the degree $d$ is even then

$$
\mathcal{J}_{U}=\overline{\mathcal{Z}_{\mathcal{C}_{d+1}}} \cap U
$$


Proof. We first prove the statement for general degrees $d$. By Lemmas 2.7 and 2.8 we obtain the inclusion

$$
\mathcal{J}_{U} \cup\left(\mathcal{Z}_{\mathcal{C}_{d+1}} \cap U\right) \subset \overline{\mathcal{Z}_{\mathcal{C}_{d+1}}} \cap U
$$

Therefore it suffices to show that every $\lambda \in \mathcal{F}_{U}$ either is an isolated point of $\mathcal{Z}_{\mathcal{C}_{d+1}}$ or is not contained in $\overline{\mathcal{Z}_{\mathcal{C}_{d+1}}}$. The map $f_{\lambda}$ is hyperbolic, therefore the orbit of 1 converges to an attracting periodic point $Q_{\lambda}$ of period $N$. By Proposition 3.4 when $U=\Lambda_{1}^{\text {hyp }}$ the Fatou set $F_{\lambda}$ is connected and $N=1$. Similarly, when $U=\Lambda_{-1}^{\text {hyp }}$, the set $F_{\lambda}$ is the union of two distinct connected components, and $N=2$.

The parameter $\lambda$ is passive, therefore $f_{\mu}^{2 n+k}(1) \rightarrow f^{k}\left(Q_{\mu}\right)$ uniformly on some small ball $B(\lambda, \varepsilon)$, where $k=0,1$ and $Q_{\mu}$ is the holomorphic continuation of the periodic point $Q_{\lambda}$. The point -1 cannot be an attracting periodic point of order 1 or 2 , thus $Q_{\mu}, f_{\mu}\left(Q_{\mu}\right) \neq-1$. We conclude that whenever $n$ is sufficiently large the point $f_{\mu}^{n}(1)$ is bounded away from -1 . Therefore the intersection $B(\lambda, \varepsilon) \cap \mathcal{Z}_{U}$ only contains isolated points.

Now suppose that $d$ is even and let $\lambda \in \mathcal{Z}_{\mathcal{C}_{d+1}} \cap U$. Let $N>0$ be the first integer so that $f_{\lambda}^{N}(1)=-1$. Since $d$ is even the point -1 is periodic with period $N$. If $N>2$ the point -1 is a repelling periodic point, since attracting fixed points in $U$ have period 1 or 2 . On the other hand -1 cannot be an attracting periodic point with order 1 or 2 . This shows that $\mathcal{Z}_{\mathcal{C}_{d+1}} \cap U \subset \mathcal{J}_{U}$, which proves (8).

Proposition 3.11. Let $1<b<(d+1) /(d-1)$. Then the set $\mathcal{J}_{\Lambda_{1}^{\text {hyp }}}$ is a Cantor set, with respect to the intrinsic topology of $\Lambda_{1}^{\mathrm{hyp}}$.

Proof. Proposition 2.5 states that $\mathcal{J}_{\Lambda_{1} \text { hyp }}$ is a perfect set. Therefore we only need to show that every connected component $K$ of this set consists of a single point. Let $\varphi_{\mu}$ be the holomorphic motion of $J_{1}$ over $\left(\Lambda_{1}^{\text {hyp }}, 1\right)$. By Theorem 2.1 the map $\varphi_{\mu}^{-1}(1)$ is continuous and sends $K$ inside $J_{1}$. Since $J_{1}$ is a Cantor set we conclude that $\varphi_{\mu}^{-1}(1)$ is constant on $K$, and therefore that $\varphi_{\mu}(c)=1$ for some $c \in \widehat{\mathbb{C}}$ and every $\mu \in K$.

If $K$ contains more than one point then by the identity principle we must have $\varphi_{\mu}(c)=1$ for all $\mu \in U$, and in particular $c=\varphi_{1}(c)=1$, thus showing that $\Lambda_{1}^{\text {hyp }}$ is an exceptional component, contradicting Lemma 3.9. We conclude that $K$ is a single point.

Combining the previous proposition with Corollary 3.10, we conclude the proof of claim (2) in Theorem 1.4.

Now let us consider $b \geq(d+1) /(d-1)$. For every $\lambda$ with $|\lambda|=1$ the map $f_{\lambda}$ is expanding on the unit circle, even uniformly expanding when $\lambda \neq 1$ or when $b>$ $(d+1) /(d-1)$, and the Julia set equals the unit circle. Thus all $\lambda$ on the unit circle, except perhaps $\lambda=1$, are contained in a hyperbolic component, hence the Julia set varies holomorphically. The preimages of -1 lie dense on the unit circle by the uniform expansion. Observing that the hyperbolic component is not exceptional it therefore follows that +1 is a preimage of -1 for a dense set of parameters $\lambda$. We have therefore proved the first statement in Theorem 1.4. 

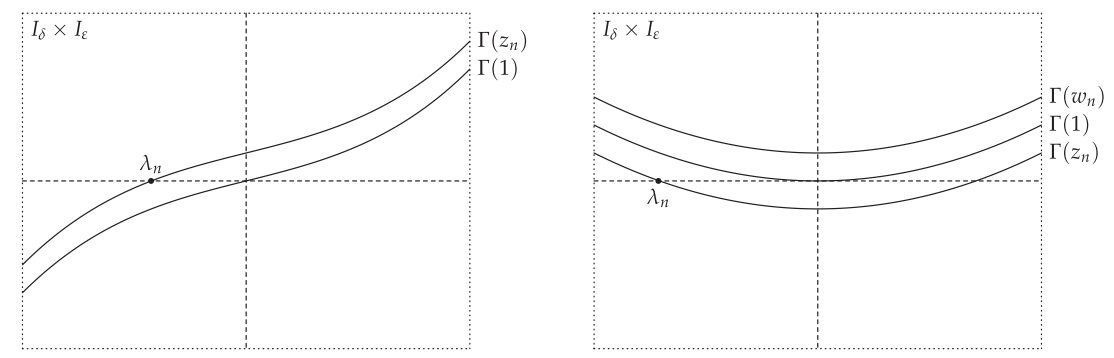

FIGURE 3. The graphs of the holomorphic motions respectively in case (1) and (2) of Lemma 4.1.

\section{Restriction to the unit circle}

Throughout this section it will be assumed that $b>1$.

LEMMA 4.1. Let $\lambda \in \mathbb{S}^{1} \cap \Lambda^{\mathrm{hyp}}$, and let $\varphi_{\mu}$ be the holomorphic motion of $J_{\lambda}$ over $\left(\Lambda_{\lambda}^{\text {hyp }}, \lambda\right)$. Assume that $1 \in J_{\lambda}$ and that one of the two following conditions is satisfied:

(1) the partial derivative $\left.\partial_{\mu} \varphi_{\mu}(1)\right|_{\mu=\lambda} \neq 0$;

(2) there exist sequences $z_{n}, w_{n} \in J_{\lambda}$ both converging to 1 and satisfying $1 \in$ $\operatorname{Arc}\left(z_{n}, w_{n}\right)$.

Then there exists a sequence $\lambda_{n} \in \mathbb{S}^{1}$ converging to $\lambda$ so that 1 is a repelling periodic point for each map $f_{\lambda_{n}}$.

See Figure 3 for an illustration.

Proof. Given $\lambda \in \mathbb{S}^{1}$, the Julia set $J_{\lambda}$ is contained in the unit circle. Therefore for every $\varepsilon>0$ there exists $\delta>0$ so that the map $\mu \mapsto \varphi_{\mu}(1)$ sends $I_{\delta}=B(\lambda, \delta) \cap \mathbb{S}^{1}$ inside a relatively compact subset of $I_{\varepsilon}=B(1, \varepsilon) \cap \mathbb{S}^{1}$. By continuity of the holomorphic motion we may assume that the same is true for the map $\mu \mapsto \varphi_{\mu}(z)$ whenever $z \in J_{\lambda}$ is sufficiently close to 1 . The map $\varphi_{\mu}(z): I_{\delta} \rightarrow I_{\varepsilon}$ can be interpreted as a map between intervals. We will denote its graph by $\Gamma(z) \subset I_{\delta} \times I_{\varepsilon}$.

Assume first that condition (1) holds. Let $z_{n} \in J_{\lambda}$ be a sequence of repelling periodic points so that $z_{n} \rightarrow 1$ and $z_{n} \neq 1$. Since $\left.\partial_{\mu} \varphi_{\mu}(1)\right|_{\mu=\lambda} \neq 0$ the graph $\Gamma(1)$ intersects the line $w=1$ transversally at the point $(\lambda, 1)$. By Theorem 2.1 , when $n$ is sufficiently large the graph $\Gamma\left(z_{n}\right)$ is uniformly close to $\Gamma(1)$ and therefore it intersects the line $w=1$ in $\left(\lambda_{n}, 1\right)$ for some $\lambda_{n} \in I_{\delta} \backslash\{\lambda\}$ close to $\lambda$. It follows that $\lambda_{n} \rightarrow \lambda$ and that $1=\varphi_{\lambda_{n}}\left(z_{n}\right)$ is a repelling periodic point for $f_{\lambda_{n}}$

Assume now that condition (2) holds. Since repelling periodic points are dense in the Julia set, we may assume from the beginning that the $z_{n}$ and $w_{n}$ are repelling periodic points for $f_{\lambda}$. When $n$ is sufficiently large the graphs $\Gamma\left(z_{n}\right)$ and $\Gamma\left(w_{n}\right)$ are both close to $\Gamma(1)$. Furthermore, since the map $z \mapsto \varphi_{\mu}(z)$ is injective, we conclude that

$$
\varphi_{\mu}(1) \in \operatorname{Arc}\left(\varphi_{\mu}\left(z_{n}\right), \varphi_{\mu}\left(w_{n}\right)\right) \text { for all } \mu \in I_{\delta},
$$

meaning that the graph $\Gamma(1)$ lies in between $\Gamma\left(z_{n}\right)$ and $\Gamma\left(w_{n}\right)$.

It follows that when $n$ is sufficiently large there exists $\lambda_{n} \in I_{\delta} \backslash\{\lambda\}$ close to $\lambda$ so that either $\varphi_{\lambda_{n}}\left(z_{n}\right)=1$ or $\varphi_{\lambda_{n}}\left(w_{n}\right)=1$. As in the previous case we obtain that 1 is a repelling periodic point for $f_{\lambda_{n}}$ and that $\lambda_{n} \rightarrow \lambda$, concluding the proof of the proposition. 
PRoposition 4.2. Let $\lambda \in \mathbb{S}^{1}$ be so that 1 is a repelling periodic point for $f_{\lambda}$. Then $\lambda \in \overline{\mathcal{Z}_{\mathcal{C}_{d+1}} \cap \mathbb{S}^{1}}$.

This proposition is proved after Lemma 4.5. We claim that it is enough to prove the statement for hyperbolic parameters. When $b>(d+1) /(d-1)$, by Proposition 3.3 all points in the circle are hyperbolic, and the claim is certainly true. Assume instead that $1<b \leq(d+1) /(d-1)$ and that the proposition holds for hyperbolic parameters. Given $\lambda \in \Lambda_{-1}^{\text {hyp }} \cap \mathbb{S}^{1}$, by Corollary 3.4 the Julia set $J_{\lambda}$ coincides with the unit circle, and by Lemma 4.1 we may find parameters in $\Lambda_{-1}^{\text {hyp }} \cap \mathbb{S}^{1}$ arbitrarily close to $\lambda$ for which 1 is a repelling periodic point. It follows that $\lambda \in \overline{\mathcal{Z}_{\mathcal{C}_{d+1}} \cap \mathbb{S}^{1}}$ and that

$$
\overline{\Lambda_{-1}^{\text {hyp }} \cap \mathbb{S}^{1}} \subseteq \overline{\mathcal{Z}_{\mathcal{C}_{d+1}} \cap \mathbb{S}^{1}} \text {. }
$$

This shows that the proposition holds also when $\lambda \in \mathbb{S}^{1}$, proving the claim (by Proposition 3.3, non-hyperbolic points on the circle are in $\overline{\Lambda_{-1}^{\text {hyp }} \cap \mathbb{S}^{1}}$ ).

From now on we will fix $\lambda \in \mathbb{S}^{1} \cap \Lambda^{\text {hyp }}$ so that 1 is a repelling point for $f_{\lambda}$. Given such $\lambda$, we will write $N$ for the period of the point 1 , and $\varphi_{\mu}$ for the holomorphic motion of $J_{\lambda}$ $\operatorname{over}\left(\Lambda_{\lambda}^{\text {hyp }}, \lambda\right)$.

Lemma 4.3. Suppose that $b \geq(d+1) /(d-1)$ or that $N \geq 3$. Then there exist sequences $z_{n}, w_{n} \in J_{\lambda}$ both converging to 1 and satisfying $1 \in \operatorname{Arc}\left(z_{n}, w_{n}\right)$.

Proof. Suppose first that $b \geq(d+1) /(d-1)$ or that $1<b<(d+1) /(d-1)$ and $\lambda \in$ $\operatorname{Arc}\left(\lambda_{1}, \bar{\lambda}_{1}\right)$. In this case $J_{\lambda}=\mathbb{S}^{1}$, and the result follows immediately.

When instead $1<b<(d+1) /(d-1)$ and $\lambda \in \operatorname{Arc}\left(\bar{\lambda}_{1}, \lambda_{1}\right)$, by Proposition 3.4 the Julia set $J_{\lambda} \subset \mathbb{S}^{1}$ is a Cantor set. Suppose for the purpose of contradiction that the lemma is false, so that $1 \in \partial \widehat{I}$, where $\widehat{I}$ is a connected component of $F_{\lambda} \cap \mathbb{S}^{1}$. The connected components of $F_{\lambda} \cap \mathbb{S}^{1}$ are open arcs which are mapped one to another by $f_{\lambda}$ and are eventually mapped into the invariant $\operatorname{arc} I_{\lambda}$ containing the unique attracting fixed point $R_{\lambda}$.

If $1 \in \partial \widehat{I}$ it follows that for some $n>0$ we must have $f_{\lambda}^{n}(1) \in \partial I_{\lambda}$. But $I_{\lambda}$ is invariant and we are assuming that 1 is periodic, therefore $1 \in \partial I_{\lambda}$. However, this is not possible when $N \geq 3$ since the boundary points of $I_{\lambda}$ are repelling periodic points with period 2 , giving a contradiction.

The point 1 is a fixed point if and only if $\lambda=1$. If $1<b<(d+1) /(d-1)$ then the point 1 is an attracting fixed point for $f_{1}$, thus if 1 is a repelling periodic point for $f_{\lambda}$ we must have $N \geq 2$.

Lemma 4.4. Suppose that $1<b<(d+1) /(d-1)$ and that $N=2$. Then $\left.\partial_{\mu} \varphi_{\mu}(1)\right|_{\mu=\lambda} \neq 0$.

Proof. Suppose instead that $\left.\partial_{\mu} \varphi_{\mu}(1)\right|_{\mu=\lambda}=0$ and write $f_{\lambda}(z)=\lambda g(z)$. Since the point $\varphi_{\mu}(1)$ is a repelling periodic point of period 2 for $f_{\mu}$, it follows that

$$
\begin{aligned}
0 & =\left.\partial_{\mu}\left(f_{\mu}^{2} \circ \varphi_{\mu}(1)\right)\right|_{\mu=\lambda} \\
& =g(\lambda)+f_{\lambda}^{\prime}(\lambda) .
\end{aligned}
$$


For $\lambda \in \mathbb{S}^{1}$ we then have $\left|f_{\lambda}^{\prime}(\lambda)\right|=|g(\lambda)|=1$, and therefore $\left|\left(f_{\lambda}^{2}\right)^{\prime}(1)\right|=\left|f_{\lambda}^{\prime}(1)\right|<1$, contradicting the fact that 1 is a repelling fixed point with period 2 .

Since $\lambda$ is a hyperbolic parameter, there exist an integer $j \geq 1$, an open neighbourhood $U \supset J_{\lambda}$ and $\kappa>1$ so that whenever $z, w \in U$ are sufficiently close we have

$$
\left|f_{\lambda}^{j N}(z)-f_{\lambda}^{j N}(w)\right| \geq \kappa|z-w| .
$$

Given $\delta>0$ sufficiently small, we may further assume that the same is true for $f_{\mu}$ when we take $\mu \in I_{\delta}=B(\lambda, \delta) \cap \mathbb{S}^{1}$. Since the map $\mu \mapsto J_{\mu}$ is continuous with respect to the Hausdorff distance, we may further assume that $J_{\mu} \subset U$ for every $\mu \in I_{\delta}$. We therefore obtain the following lemma.

LEMMA 4.5. There exists $\varepsilon>0$ so that for every $\mu \in I_{\delta}$ and $z, w \in J_{\mu}$ distinct we can find $k \geq 0$ so that

$$
\left|f_{\mu}^{k N}(z)-f_{\mu}^{k N}(w)\right| \geq 2 \varepsilon .
$$

Proof of Proposition 4.2. Let $\lambda \in \Lambda^{\text {hyp }} \cap \mathbb{S}^{1}$ be a parameter for which 1 is a repelling periodic point of period $N \geq 2$. We will assume first that $b \geq(d+1) /(d-1)$ or that $N \geq 3$.

By Lemma 4.3 there exist two sequences in $J_{\lambda}$ converging to 1: one contained in the upper half plane and one in the lower half plane. Since the backward images of the point -1 accumulate on the Julia set $J_{\lambda}$, and thus on every point in such sequences, we may find two preimages $\alpha_{\lambda}, \beta_{\lambda}$ of the point -1 contained in $I_{\varepsilon}=B(1, \varepsilon) \cap \mathbb{S}^{1}$ so that $1 \in \operatorname{Arc}\left(\alpha_{\lambda}, \beta_{\lambda}\right)$. Write $M_{1}, M_{2}>0$ for the two positive integers so that $f^{M_{1}}\left(\alpha_{\lambda}\right)=f^{M_{2}}\left(\beta_{\lambda}\right)=-1$.

Take $\delta>0$ and write $I_{\delta}=B(\lambda, \delta) \cap \mathbb{S}^{1}$. Then if $\delta$ is sufficiently small we have $\varphi_{\mu}(1)$ : $I_{\delta} \rightarrow I_{\varepsilon}$ and we can find two continuous functions $\alpha_{\mu}, \beta_{\mu}: I_{\delta} \rightarrow I_{\varepsilon}$ so that $f^{M_{1}}\left(\alpha_{\mu}\right)=$ $f^{M_{2}}\left(\beta_{\mu}\right)=-1$ and

$$
\varphi_{\mu}(1) \in \operatorname{Arc}\left(\alpha_{\mu}, \beta_{\mu}\right) \quad \text { for all } \mu \in I_{\delta} \text {. }
$$

Lemma 4.3 shows that condition (2) in Lemma 4.1 is satisfied. It follows that there exists $\lambda^{\prime} \in I_{\delta} \backslash\{\lambda\}$ arbitrarily close to $\lambda$ so that 1 is also a repelling periodic point for $f_{\lambda^{\prime}}$, and therefore $1 \in J_{\lambda^{\prime}}$. Furthermore, by Lemma 3.9 the holomorphic map $\varphi_{\mu}(1)$ is not constant, therefore we may choose $\lambda^{\prime}$ so that $1 \neq \varphi_{\lambda^{\prime}}(1)$.

By Lemma 4.5 we may find $k$ so that $\left|f_{\lambda^{\prime}}^{k N}\left(\varphi_{\lambda^{\prime}}(1)\right)-f_{\lambda^{\prime}}^{k N}(1)\right| \geq 2 \varepsilon$, and since $\varphi_{\lambda^{\prime}}(1) \in I_{\varepsilon}$ is a periodic point for $f_{\lambda^{\prime}}$ of period $N$ we conclude that

$$
\left|f_{\lambda^{\prime}}^{k N}(1)-1\right| \geq\left|f_{\lambda^{\prime}}^{k N}(1)-\varphi_{\lambda^{\prime}}(1)\right|-\left|\varphi_{\lambda^{\prime}}(1)-1\right| \geq \varepsilon,
$$

showing that $f_{\lambda^{\prime}}^{k N}(1) \in \mathbb{S}^{1} \backslash I_{\varepsilon}$.

The map $\mu \mapsto f_{\mu}^{k N}(1)$ is continuous and we have

$$
f_{\lambda}^{k N}(1)=1 \in \operatorname{Arc}\left(\alpha_{\lambda}, \beta_{\lambda}\right), \quad f_{\lambda^{\prime}}^{k N}(1) \notin \operatorname{Arc}\left(\alpha_{\lambda^{\prime}}, \beta_{\lambda^{\prime}}\right) .
$$

Therefore we may conclude that there exists $\lambda^{\prime \prime} \in \operatorname{Arc}\left(\lambda, \lambda^{\prime}\right)$ so that either $f_{\lambda^{\prime \prime}}^{k N}(1)=\alpha_{\lambda^{\prime \prime}}$ or $f_{\lambda^{\prime \prime}}^{k N}(1)=\beta_{\lambda^{\prime \prime}}$, see Figure 4 for an illustration of the points $\lambda^{\prime}$ and $\lambda^{\prime \prime}$. Since $\alpha_{\lambda^{\prime \prime}}$ and $\beta_{\lambda^{\prime \prime}}$ are preimages of the point -1 we conclude in both cases that $\lambda^{\prime \prime} \in \mathcal{Z}_{\mathcal{C}_{d+1}} \cap \mathbb{S}^{1}$. Since $\lambda^{\prime}$, and thus $\lambda^{\prime \prime}$, can be chosen arbitrarily close to $\lambda$, we must have $\lambda \in \overline{\mathcal{Z}_{\mathcal{C}_{d+1}} \cap \mathbb{S}^{1}}$. 


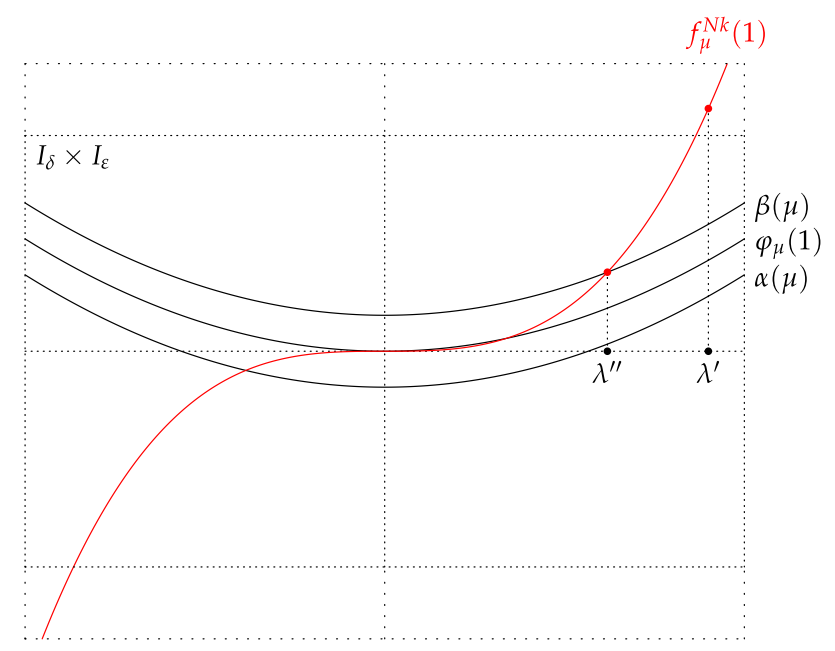

FIGURE 4. The position of the points $\lambda^{\prime}$ and $\lambda^{\prime \prime}$.

Suppose now that $1<b<(d+1) /(d-1)$ and that $N=2$. We notice that once $b$ and $d$ are fixed, this can happen only for finitely many values of $\lambda$. Combining Lemma 4.4 and Lemma 4.1. we can find $\lambda^{\prime} \in \Lambda^{\text {hyp }} \cap \mathbb{S}^{1}$ arbitrarily close to $\lambda$ for which 1 is a repelling fixed point for $f_{\lambda^{\prime}}$ with period greater than 3 . Since the proposition holds for $\lambda^{\prime}$ it must hold for $\lambda$ as well, concluding the proof of the proposition.

Proof of claim (1) in Theorem 1.4. We already showed that Lemma 4.1 and Proposition 4.2 together imply $\overline{\Lambda_{-1}^{\text {hyp }} \cap \mathbb{S}} \subset \overline{\mathcal{Z}_{\mathcal{C}_{d+1}} \cap \mathbb{S}}$. Therefore when $1<b<(d+1) /(d-1)$, by Proposition 3.3 we have

$$
\operatorname{Arc}\left[\lambda_{1}, \overline{\lambda_{1}}\right] \subset \overline{\mathcal{Z}_{\mathcal{C}_{d+1}} \cap \mathbb{S}} .
$$

If $\lambda=\lambda_{0}$ or $\lambda=\overline{\lambda_{0}}$, then 1 is a repelling 2-cycle of $f_{\lambda_{0}}$, therefore by Proposition 4.2 we have $\left\{\lambda_{0}, \overline{\lambda_{0}}\right\} \subset \overline{\mathcal{Z}_{\mathcal{C}_{d+1}} \cap \mathbb{S}}$.

\section{Hyperbolic semigroups and expanding orbits}

Throughout this section we will assume that $d \in \mathbb{N}_{\geq 2}$ and $b \in(1,(d+1) /(d-1))$ are fixed.

Definition 5.1. Given $\lambda \in \hat{\mathbb{C}}$, we define the semigroup $H=\left\langle f_{1}, \ldots, f_{d}\right\rangle$ as the semigroup generated by the maps

$$
f_{k}(z)=\lambda\left(\frac{z+b}{b z+1}\right)^{k}, \quad k=1, \ldots, d .
$$

We will write $F_{k}, J_{k}$ for the Fatou and Julia set of the map $f_{k}$ and $F_{H}, J_{H}$ for the Fatou and Julia set of the semigroup $H$.

For most values of $\lambda$ we can give the following precise characterization of the semigroup $H$, which will not be used later in the paper but may be interesting in its own right. 
PROPOSITION 5.2. For all but countably many $\lambda \in \widehat{\mathbb{C}}$ the semigroup $H$ is freely generated by $\left\{f_{1}, \ldots, f_{d}\right\}$.

Proof. Let $S$ be the free group generated by $\left\{f_{1}, \ldots, f_{d}\right\}$ and write $\Phi: S \rightarrow H$ for the homomorphism

$$
\Phi\left[f_{i_{1}} \cdots f_{i_{k}}\right]=f_{i_{k}} \circ \cdots \circ f_{i_{1}} .
$$

Given a word $s=f_{i_{1}} \cdots f_{i_{k}} \in S$, the map $\Phi[s]$ is a rational map in both $z$ and $\lambda$. Its degrees with respect to $z$ and $\lambda$ are equal to

$$
\begin{aligned}
\operatorname{deg}_{z}(s) & =i_{1} \cdots i_{k}, \\
\operatorname{deg}_{\lambda}(s) & =1+i_{k}+i_{k} \cdot i_{k-1}+\cdots+i_{k} \cdots i_{1} .
\end{aligned}
$$

We notice that the map $\Phi[s](1)$ is also a rational map in $\lambda$ of degree

$$
\operatorname{deg}_{\lambda}^{\prime}(s)=1+i_{k}+i_{k} \cdot i_{k-1}+\cdots i_{k} \cdots i_{2} .
$$

The set $S$ is countable, therefore it is sufficient to show that for every $s_{1}, s_{2} \in S$ either $\Phi\left(s_{1}\right)=\Phi\left(s_{2}\right)$ for finitely many $\lambda$ or $s_{1}=s_{2}$.

Suppose instead that $s_{1}=f_{i_{1}} \cdots f_{i_{k}}$ and $s_{2}=f_{j_{1}} \cdots f_{j_{l}}$ satisfy $\Phi\left(s_{1}\right)=\Phi\left(s_{2}\right)$ for infinitely many $\lambda$. Using the identity principle, it is not hard to show that $\Phi\left(s_{1}\right)(z)=$ $\Phi\left(s_{2}\right)(z)$ holds for all $\lambda \in \widehat{\mathbb{C}}$. This shows that the maps $\Phi\left(s_{1}\right)$ and $\Phi\left(s_{2}\right)$ coincide as rational maps in both variables $z$ and $\lambda$, and therefore that

$$
i_{1}=\frac{\operatorname{deg}_{z}\left(s_{1}\right)}{\operatorname{deg}_{\lambda}\left(s_{1}\right)-\operatorname{deg}_{\lambda}^{\prime}\left(s_{1}\right)}=\frac{\operatorname{deg}_{z}\left(s_{2}\right)}{\operatorname{deg}_{\lambda}\left(s_{2}\right)-\operatorname{deg}_{\lambda}^{\prime}\left(s_{2}\right)}=j_{1} .
$$

If we now write $s_{1}^{\prime}=f_{i_{2}} \cdots f_{i_{k}}$ and $s_{2}^{\prime}=f_{j_{2}} \cdots f_{j_{l}}$ we obtain that $\Phi\left(s_{1}^{\prime}\right)=\Phi\left(s_{2}^{\prime}\right)$ also holds for infinitely many $\lambda$ and therefore $i_{2}=j_{2}$. Iterating this procedure, we obtain that $s_{1}=s_{2}$, concluding the proof of the proposition.

Notice that for $k \leq d$ we have $(k+1) /(k-1) \geq(d+1) /(d-1)$ and hence $b \in$ $(1,(k+1) /(k-1)$.

Notation 5.3. For simplicity of notation we are avoiding (where possible) the use of the subscript $\lambda$. As an example, notice that we are writing $f_{k}$ instead of the more accurate $f_{k, \lambda}$. The function $f_{d}$ will play an important role in the analysis of the semigroup $H$. Therefore we will write $\lambda_{0}$ and $\lambda_{1}$ for the parameters obtained by Theorem 3.7 applied to the map $f_{d}$, and $I$ for the immediate attracting arc relative to the function $f_{d}$ (if it exists). When it is necessary to distinguish between different values of the parameter $\lambda$ we will write $f_{k, \lambda}$ and $I_{\lambda}$.

We will write $\Omega$ for the set of all possible sequences with entries in $\{1, \ldots, d\}$. For every element $g \in H$ we can find $\omega \in \Omega$ and $n \in \mathbb{N}$ so that $g=f_{\omega}^{n}$, where we write

$$
f_{\omega}^{n}=f_{\omega_{n}} \circ \cdots \circ f_{\omega_{1}} .
$$

For $0 \leq m \leq n$ we will further write

$$
f_{\omega}^{n, m}=f_{\omega_{n}} \circ \cdots \circ f_{\omega_{m}} .
$$


Definition 5.4. We define the length of an element $g \in H$ as the minimum integer $n$ for which $g=f_{\omega}^{n}$ for some $\omega \in \Omega$.

We proved in $\S 4$ that for any $\lambda$ in the closed $\operatorname{arc} \operatorname{Arc}\left[\lambda_{1},-1\right]$ there exist a (rooted) Cayley tree $T_{n}$ of degree $d$ and a parameter $\lambda^{\prime} \in \mathbb{S}^{1}$ arbitrarily close to $\lambda$ so that

$$
Z_{T_{n}}\left(\lambda^{\prime}, b\right)=0
$$

Recall from [PR20, Theorem B], on the other hand, that for any $\lambda \in\left[1, \lambda_{0}\right)$, any $r \geq 0$ and any graph $G$ of degree $d$ we have

$$
Z_{G}(r \cdot \lambda, b) \neq 0 .
$$

The situation for the $\operatorname{arc} \operatorname{Arc}\left(\lambda_{0}, \lambda_{1}\right)$ appears to be more complicated. If we only consider Cayley trees of degree $d$ it is possible to show that there indeed exist zeros of the partition function of some tree $T_{n}$ on the $\operatorname{arc} \operatorname{Arc}\left(\lambda_{0}, \lambda_{1}\right)$. However, as shown in $\S 3$, these zeros form a nowhere dense subset of the arc. Our purpose is to show that zeros of the partition for general bounded degree graphs are dense in $\operatorname{Arc}\left[\lambda_{0}, \lambda_{3}\right]$, where $\lambda_{3}$ is a parameter on the unit circle close to $\lambda_{0}$. In order to do so we will consider the class of rooted spherically symmetric trees of bounded degree for which, according to Lemma 1.6, the zero parameters can be understood by studying the semigroup dynamics of $H$.

In what follows we will study the semigroup dynamics (mostly) under the assumption that $\lambda \in \mathbb{S}^{1}$. Under these assumptions for any $g \in H_{\lambda}$ we have $g\left(\hat{\mathbb{C}} \backslash \mathbb{S}^{1}\right)=\hat{\mathbb{C}} \backslash \mathbb{S}^{1}$, and therefore that

$$
\hat{\mathbb{C}} \backslash \mathbb{S}^{1} \subset F_{H_{\lambda}}, \quad J_{H_{\lambda}} \subset \mathbb{S}^{1}
$$

\subsection{Hyperbolicity of the semigroup.}

Lemma 5.5. There exists $\lambda_{2} \in \operatorname{Arc}\left(\lambda_{0}, \lambda_{1}\right)$ so that for every $\lambda \in \operatorname{Arc}\left[\lambda_{0}, \lambda_{2}\right)$ and every $k \in\{1, \ldots, d-1\}$ we have

$$
f_{1} \circ f_{k}(I) \Subset I .
$$

Proof. Assume first that $\lambda=\lambda_{0}$ and recall that by Theorem 3.7 we have $I=\operatorname{Arc}\left(1, \lambda_{0}\right)$. The Möbius transformation $\gamma(z)=(z+b) /(b z+1)$ is a bijection of the unit circle into itself which reverses the orientation, and $f_{k}(z)=\lambda_{0} \gamma(z)^{k}$. The map $f_{d}: I \rightarrow I$ is an orientation-reversing bijection and therefore satisfies $f_{d}(I)=I$. It follows immediately that, if we write $\ell$ for the length of the arc $I$, the image $f_{k}(I)$ is an $\operatorname{arc}$ of length $\ell^{k / d}<\ell$, therefore the map $f_{k}: I \rightarrow \mathbb{S}^{1}$ is not surjective.

Notice that when the point $z$ moves counterclockwise on the $\operatorname{arc} I=\operatorname{Arc}\left(1, \lambda_{0}\right)$ starting at the point 1 , its image $f_{k}(z)$ moves clockwise on the unit circle, starting at $\lambda_{0}=f_{k}(1)$, until it reaches $f_{k}\left(\lambda_{0}\right)$. In principle it is possible that $f_{k}(z)$ rotates once or more times around the circle, but since $f_{k}$ is not surjective on $I$ this does not happen. We conclude that $f_{k}: I \rightarrow \operatorname{Arc}\left(f_{k}\left(\lambda_{0}\right), \lambda_{0}\right)$ is also an orientation-reversing bijection, and since the length of the arc $f_{k}(I)$ is less than the length of $I$, we must have $f_{k}\left(\lambda_{0}\right) \in I$. If we now compose 
with the map $f_{1}$ we find that

$$
f_{1} \circ f_{k}(I)=f_{1}\left(\operatorname{Arc}\left(f_{k}\left(\lambda_{0}\right), \lambda_{0}\right)\right)=\operatorname{Arc}\left(f_{1}\left(\lambda_{0}\right), f_{1} \circ f_{k}\left(\lambda_{0}\right)\right) \Subset I .
$$

Since $I$ moves continuously in $\lambda$, the same holds sufficiently close $\lambda_{0}$, which implies the existence of $\lambda_{2}$.

In the following we will denote by $\lambda_{2}$ the parameter with the maximal argument that satisfies the requirements of the previous lemma.

Definition 5.6. We define the semigroup $\widehat{H} \subset H$ as the semigroup generated by the maps

$$
\widehat{H}=\left\langle\widehat{f_{1}}, \ldots, \widehat{f_{d}}\right\rangle,
$$

where $\widehat{f}_{d}=f_{d}$ and $\widehat{f_{k}}=f_{1} \circ f_{k}$ for $k \in\{1, \ldots, d-1\}$. We will write $F_{\widehat{H}}, J_{\widehat{H}}$ for the Fatou and the Julia set of the semigroup $\widehat{H}$.

Since $F_{H} \subset F_{\widehat{H}}$, it follows that $\widehat{\mathbb{C}} \backslash \mathbb{S}^{1} \subset F_{\widehat{H}}$. Furthermore, by the previous lemma the interval $I$ is invariant for every map in $\widehat{H}$ for $\lambda \in \operatorname{Arc}\left[\lambda_{0}, \lambda_{2}\right.$ ), and therefore it is contained in $F_{\widehat{H}}$, proving that for these $\lambda$,

$$
\left(\widehat{\mathbb{C}} \backslash \mathbb{S}^{1}\right) \cup I \subset F_{\widehat{H}}, \quad J_{\widehat{H}} \subset \mathbb{S}^{1} \backslash I,
$$

and therefore that the Fatou set $F_{\widehat{H}}$ is connected.

Similarly to the case of the semigroup $H$, given $\omega \in \Omega$ and $0 \leq m \leq n$, we will write

$$
\begin{aligned}
\widehat{f}_{\omega}^{n} & =\widehat{f}_{\omega_{n}} \circ \cdots \circ \widehat{f}_{\omega_{1}}, \\
\widehat{f}_{\omega}^{n, m} & =\widehat{f}_{\omega_{n}} \circ \cdots \circ \widehat{f}_{\omega_{m}} .
\end{aligned}
$$

Also in this case every element of the semigroup can be written as $\widehat{f}_{\omega}^{n}$ for some $\omega \in \Omega$ and $n \in \mathbb{N}$.

By the previous lemma, given $\lambda \in \operatorname{Arc}\left[\lambda_{0}, \lambda_{2}\right)$, it follows that there exists a closed arc $J \subset I$ so that $\widehat{f_{k}}(J) \Subset J$ for every $k=1, \ldots, d$. Indeed, if we write $I=\operatorname{Arc}(z, w)$, this property is satisfied by $J=\operatorname{Arc}[\zeta, \eta]$, where $\zeta$ is sufficiently close to $z$ and $\eta$ lies in $\operatorname{Arc}\left(f_{d}(\zeta), f_{d}^{-1}(\zeta)\right)$ (where the preimage is taken inside $I$ ). We may therefore define the family $\mathcal{C}$ and the closed $\operatorname{arc} K$ as follows:

$$
\begin{aligned}
\mathcal{C} & :=\left\{J \subset I \mid J \neq \emptyset \text { closed arc such that } \widehat{f_{k}}(J) \Subset \operatorname{int}(J) \text { for all } k=1, \ldots, d\right\}, \\
K & :=\bigcap_{J \in \mathcal{C}} J .
\end{aligned}
$$

In this definition $\operatorname{int}(J)$ refers to the interior of $J$ with respect to the topology of the unit circle.

LemmA 5.7. Let $\lambda \in \operatorname{Arc}\left[\lambda_{0}, \lambda_{2}\right)$. Then the set $K \subset I$ is a non-empty closed arc which is forward invariant under $\widehat{H}$. Furthermore, the map $\lambda \mapsto K_{\lambda}$ is upper semicontinuous for $\lambda \in \operatorname{Arc}\left[\lambda_{0}, \lambda_{2}\right)$. 
Proof. Write $\mathcal{C}_{\mathbb{Q}}$ for the subset of all intervals $J \in \mathcal{C}$ whose extrema are rational angles. Notice that, given $J \in \mathcal{C}$, there exists $J^{\prime} \in \mathcal{C}_{\mathbb{Q}}$ for which $J^{\prime} \subset J$, and therefore

$$
K=\bigcap_{J \in \mathcal{C}_{\mathbb{Q}}} J .
$$

Given $J \in \mathcal{C}$, we have that $f_{d}(J) \Subset J$ and $J \subset I$, proving that the arc $J$ contains the unique attracting fixed point of $f_{d}$. This shows that for any pair $J_{1}, J_{2} \in \mathcal{C}$ the intersection $J^{\prime}=J_{1} \cap J_{2}$ is non-empty. Furthermore, one can show that $J^{\prime}$ is again an element of $\mathcal{C}$. Similarly, given $J_{1}, J_{2} \in \mathcal{C}_{\mathbb{Q}}$, one has that $J_{1} \cap J_{2} \in \mathcal{C}_{\mathbb{Q}}$.

The set $\mathcal{C}_{\mathbb{Q}}$ is countable, hence we can enumerate its elements as $\left\{J_{k}\right\}_{k \geq 1}$. If we write $L_{k}=J_{1} \cap \cdots \cap J_{k}$ it then follows that $L_{k} \in \mathcal{C}_{\mathbb{Q}}$, that $L_{k+1} \subset L_{k}$ and that

$$
K=\bigcap_{k=1}^{\infty} L_{k} .
$$

This shows that $K$ is the intersection of a nested family of non-empty, compact and connected arcs, and therefore that $K$ is a non-empty closed arc. Since every $L_{k}$ is forward invariant for any $g \in \widehat{H}$, the same holds for $K$.

We will now write $\mathcal{C}_{\mu}, K_{\mu}$ in order to study parameter values close to $\lambda$. On the other hand, we will write $L_{k}$ for the set defined above for the parameter value $\lambda$. For every positive integer $k$ we have $L_{k} \in \mathcal{C}_{\mu}$ for every $\mu \in \operatorname{Arc}\left[\lambda_{0}, \lambda_{2}\right)$ sufficiently close to $\lambda$, and for such parameters $\mu$ it then follows that $K_{\mu} \subset L_{k}$. Since $L_{k}$ is a sequence of nested sets approximating $K_{\lambda}$, we conclude immediately that the map $\mu \mapsto K_{\mu}$ is upper semicontinuous at the point $\lambda$, concluding the proof of the lemma.

Proposition 5.8. Let $\lambda \in \operatorname{Arc}\left[\lambda_{0}, \lambda_{2}\right)$. Then every limit of a convergent sequence in $\widehat{H}$ with divergent length is constant on $F_{\widehat{H}}$ and contained in $K$.

Proof. Let $g_{k} \in \widehat{H}$ be a sequence with divergent length that converges uniformly to $g_{\infty}$ : $F_{\widehat{H}} \rightarrow \bar{F}_{\widehat{H}}=\hat{\mathbb{C}}$. Choose sequences $\omega_{k} \in \Omega$ and $n_{k} \in \mathbb{N}$ so that $g_{k}=\widehat{f}_{\omega_{k}}^{n_{k}}$, and notice that since the sequence $g_{k}$ has divergent length we must have $n_{k} \rightarrow \infty$. Since $F_{\widehat{H}} \cap \mathbb{S}^{1} \neq \emptyset$, it follows that $F_{\widehat{H}}$ is connected. Write $\rho$ for the hyperbolic metric of $F_{\widehat{H}}$.

The Fatou set $F_{\widehat{H}}$ is forward invariant with respect to each element in the semigroup. In particular, we have $\widehat{f_{1}}\left(F_{\widehat{H}}\right) \subset F_{\widehat{H}}$. Since the closed arc $K \subset F_{\widehat{H}}$ is forward invariant, it contains the unique attracting fixed point $R_{1}$ of $\widehat{f_{1}}$ (which coincides with the attracting fixed point of $\left.f_{1}\right)$. Assume now that $\widehat{f_{1}}\left(F_{\widehat{H}}\right)=F_{\widehat{H}}$. Since $\widehat{f}_{1}$ is invertible, it then follows that the Fatou set $F_{\widehat{H}}$ is completely invariant with respect to $\widehat{f_{1}}$, and therefore it contains the whole attracting basin of $R_{1}$. Since $\widehat{f_{1}}$ is a Möbius transformation, we conclude that the Julia set of the semigroup $J_{\widehat{H}}$ must consist of a single point, giving a contradiction. Therefore

$$
\widehat{f_{1}}\left(F_{\widehat{H}}\right) \subsetneq F_{\widehat{H}}
$$

For every $k=2, \ldots, d$ the map $\widehat{f_{k}}$ has two critical points $\{-b,-1 / b\} \subset F_{\widehat{H}}$. It follows from the Schwarz-Pick lemma that for every $k=1, \ldots, d$ the map $\widehat{f}_{k}$ is a contraction with respect to the metric $\rho$. This means that for every compact set $Q \subset F_{\widehat{H}}$ we may find 
a constant $c_{Q}<1$ so that for any $z, w \in Q$ we have

$$
\rho\left(\widehat{f_{k}}(w), \widehat{f_{k}}(z)\right) \leq c_{Q} \cdot \rho(w, z), \quad k=1, \ldots, d .
$$

Let $K$ be as in (11), $r \in \mathbb{R}_{>0}$ and define $Q=\bar{B}_{\rho}(K, r)$, where the ball is taken with respect to the hyperbolic metric of $F_{\widehat{H}}$. Given $z \in K$ and $w \in B_{\rho}(z, r)$, we know that for every $k, n \geq 0$ we have $\widehat{f}_{\omega_{k}}^{n}(z) \in K$ and

$$
\rho\left(\widehat{f}_{\omega_{k}}^{n}(w), \widehat{f}_{\omega_{k}}^{n}(z)\right) \leq \rho(w, z) .
$$

This shows that $\widehat{f}_{\omega_{k}}^{n}(w), \widehat{f}_{\omega_{k}}^{n}(z) \in Q$, and thus that

$$
\rho\left(\widehat{f}_{\omega_{k}}^{n_{k}}(w), \widehat{f}_{\omega_{k}}^{n_{k}}(z)\right) \leq c_{Q}^{n_{k}} \cdot \rho(w, z),
$$

which finally implies that $g_{\infty}(w)=g_{\infty}(z)$ for every $w \in B_{\rho}(z, r)$. We took $r$ to be arbitrary and thus we can conclude that $g_{\infty}$ is constant. For every $g \in \widehat{H}$ and $z \in K$ it follows that $g(z) \in K$, completing the proof.

We recall the following definition of hyperbolicity for semigroups, introduced in [Sum97, Sum98].

Definition 5.9. Let $G$ be a rational semigroup and consider the postcritical set

$$
P_{G}:=\overline{\bigcup_{g \in G}\{\text { critical values of } g\}}
$$

We say that the semigroup is hyperbolic if $P_{G} \subset F_{G}$.

PROPOSITION 5.10. The semigroup $\widehat{H}$ is hyperbolic.

Proof. In our case the postcritical set can be written as

$$
P_{\widehat{H}}=\overline{\bigcup_{\substack{\omega \in \Omega \\ n \in \mathbb{N}}} \widehat{f}_{\omega}^{n}(\{-b,-1 / b\})} .
$$

It is clear that $f_{\omega}^{n}(\{-b,-1 / b\}) \in \widehat{\mathbb{C}} \backslash \mathbb{S}^{1}$ for every $n$ and $\omega$. Furthermore, by the previous theorem we know that every limit point belongs to $K$, showing that

$$
P_{\widehat{H}} \subset\left(\widehat{\mathbb{C}} \backslash \mathbb{S}^{1}\right) \cup K \subset F_{\widehat{H}}
$$

Given $\omega \in \Omega$, we write $F_{\omega}, J_{\omega}$ for the Fatou and Julia sets of the family $\left\{\widehat{f}_{\omega}^{n}\right\}$. By (10) we have that

$$
J_{\omega} \subset J_{\widehat{H}} \subset \mathbb{S}^{1} \backslash I
$$

and one can show that if $z \in J_{\omega}$ its orbit avoids the set $I$. On the other hand, given $z \in$ $F_{\omega} \cap \mathbb{S}^{1}$, by Proposition 5.8 we can find $n>0$ so that $\widehat{f}_{\omega}^{n}(z) \in I$. We conclude that we can 


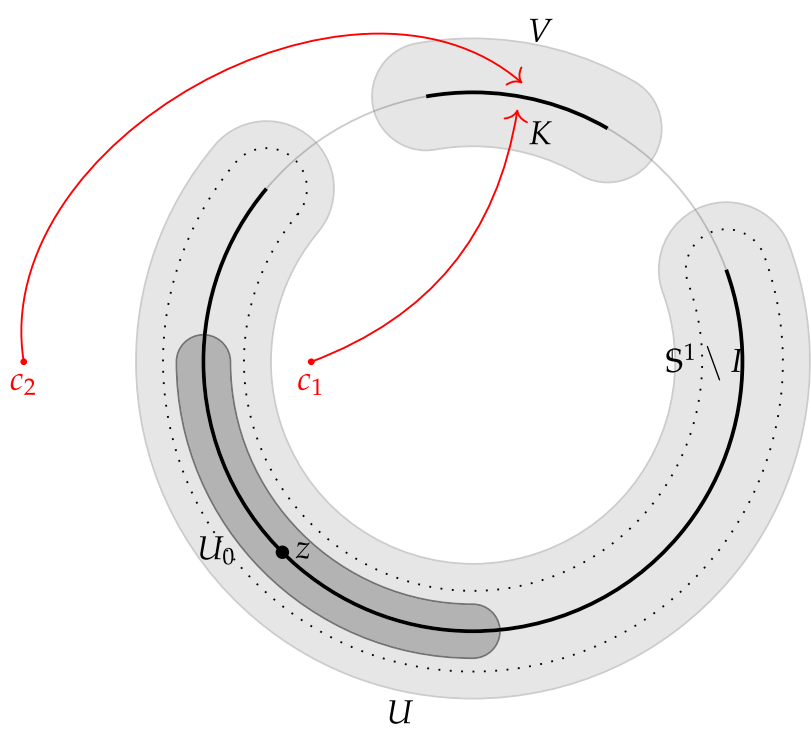

FIGURE 5. Sets used in the proof of Lemma 5.11.

write

$$
J_{\omega}=\bigcap_{n \in \mathbb{N}}\left(\widehat{f}_{\omega}^{n}\right)^{-1}\left(\mathbb{S}^{1} \backslash I\right)
$$

The following lemma shows expansivity of the dynamics on the Julia set $J_{\omega}$ of every sequence $\omega$. The result corresponds to [Sum98, Theorem 2.6]; for the sake of completeness we provide a sketch of the proof.

LEMmA 5.11. Let $\lambda \in \operatorname{Arc}\left[\lambda_{0}, \lambda_{2}\right)$ and $\kappa>1$. Then there exists a positive integer $N \geq 1$ so that for any $\omega \in \Omega$ and $z \in J_{\omega}$ we have

$$
\left|\left(\widehat{f}_{\omega}^{N}\right)^{\prime}(z)\right| \geq \kappa
$$

Sketch of the proof. We start by choosing an open simply connected neighbourhood $V \subset\left(\widehat{\mathbb{C}} \backslash \mathbb{S}^{1}\right) \cup I$ containing $K$ and two open simply connected neighbourhoods $U^{\prime} \Subset U$ containing $\mathbb{S}^{1} \backslash I$ and disjoint from $P_{\widehat{H}}$ and $V$, see Figure 5 for an illustration. Then choose $C>1$ so that $d \rho_{U^{\prime}} \geq C \cdot d \rho_{U}$, where we write $d \rho_{U}, d \rho_{U^{\prime}}$ for the infinitesimal hyperbolic metric of the two sets.

By Proposition 5.8 there exists a positive integer $N_{0}$ so that

$$
\widehat{f}_{\omega}^{N_{0}}\left(\bar{U} \backslash U^{\prime}\right) \subset V \text { for all } \omega \in \Omega \text {, for all } n \geq N_{0} .
$$

Given $\omega \in \Omega$ and $z \in J_{\omega}$, by (12), we have $\widehat{f}_{\omega}^{n}(z) \in U^{\prime}$ for every $n$. Therefore we may define $U_{0} \subset U^{\prime}$ as the connected component of $\left(\widehat{f}_{\omega}^{N_{0}}\right)^{-1}(U)$ containing $z$. Since $U$ is simply connected and disjoint from the postcritical set, the map $\widehat{f}_{\omega}^{N_{0}}$ preserves the hyperbolic metrics of $U_{0}$ and $U$. Since $U_{0} \subset U^{\prime}$, this implies that

$$
\left|\left(\widehat{f}_{\omega}^{N_{0}}\right)^{\prime}(z)\right|_{U} \geq C .
$$


The hyperbolic metric of $U$ and the Euclidean metric are comparable on $U^{\prime}$. Therefore by taking an integer $k$ sufficiently large, which does not depend on the choice of the sequence $\omega$ and $z$, we conclude that the value $N=k N_{0}$ satisfies the requirements of the lemma.

We will now show that, once we are bounded away from $\lambda_{2}$, the value of $N$ can be chosen independently of $\lambda$. It will be convenient to reintroduce the subscript $\lambda$ in order to distinguish between different parameter values. It is clear the set $J_{\omega}$ is dependent on $\lambda$, thus it will be denoted by $J_{\omega, \lambda}$.

Lemma 5.12. Let $\lambda^{\prime} \in \operatorname{Arc}\left[\lambda_{0}, \lambda_{2}\right)$ and $\kappa>1$. Then there exists a positive integer $N \geq 1$ so that for any $\lambda \in \operatorname{Arc}\left[\lambda_{0}, \lambda^{\prime}\right]$, any $\omega \in \Omega$ and any $z \in J_{\omega}$ there exists $1 \leq n \leq N$ so that

$$
\left|\left(\widehat{f}_{\omega, \lambda}^{n}\right)^{\prime}(z)\right| \geq \kappa .
$$

Proof. Given $\lambda \in \operatorname{Arc}\left[\lambda_{0}, \lambda_{2}\right)$, let $N_{\lambda}$ be the minimum integer for which the previous lemma is valid. Suppose now that there exists a sequence $\lambda_{k} \in \operatorname{Arc}\left[\lambda_{0}, \lambda^{\prime}\right]$ such that $N_{k}=$ $N_{\lambda_{k}} \rightarrow \infty$. It follows that we may find sequences $\omega_{k} \in \Omega$ and $z_{k} \in J_{\omega_{k}, \lambda_{k}}$ so that

$$
\left|\left(\widehat{f}_{\omega_{k}, \lambda_{k}}^{n}\right)^{\prime}\left(z_{k}\right)\right|<\kappa \text { for all } 0 \leq n \leq N_{k}-1
$$

By passing to a subsequence if necessary, we may assume that the three following conditions are satisfied:

(1) the parameters $\lambda_{k}$ converge to $\lambda_{\infty} \in \operatorname{Arc}\left[\lambda_{0}, \lambda_{1}\right]$;

(2) the points $z_{k}$ converge to $z_{\infty} \in \mathbb{S}^{1}$;

(3) the sequences $\omega_{k}$ and $\omega_{k+1}$ agree on the first $k$ elements.

Since the arc $I_{\lambda}$ varies continuously in $\lambda$ and by (12) every $z_{k} \in \mathbb{S}^{1} \backslash I_{\lambda_{k}}$, it follows that $z_{\infty} \in \mathbb{S}^{1} \backslash I_{\lambda_{\infty}}$. Let $\omega_{\infty}$ be the sequence given by $\omega_{\infty, k}=\omega_{k, k}$, where $\omega_{k, n}$ denotes the $n$th element of the sequence $\omega_{k}$. Then it is clear that $\omega_{k}$ and $\omega_{\infty}$ agree on the first $k$ elements. Given $n \in \mathbb{N}$, we have

$$
\widehat{f}_{\omega_{\infty}, \lambda_{\infty}}^{n}\left(z_{\infty}\right)=\lim _{k \rightarrow \infty} \widehat{f}_{\omega_{k}, \lambda_{k}}^{n}\left(z_{k}\right) \in \mathbb{S}^{1} \backslash I_{\lambda_{\infty}}
$$

proving that $z_{\infty} \in J_{\omega_{\infty}, \lambda_{\infty}}$. Furthermore, when $k$ is sufficiently large we have $n \leq N_{k}$, therefore

$$
\left|\left(\widehat{f}_{\omega_{\infty}, \lambda_{\infty}}^{n}\right)^{\prime}\left(z_{\infty}\right)\right|=\lim _{k \rightarrow \infty}\left|\left(\widehat{f}_{\omega_{k}, \lambda_{k}}^{n}\right)^{\prime}\left(z_{k}\right)\right| \leq \kappa,
$$

contradicting the previous lemma.

PROPOSITION 5.13. Let $\lambda^{\prime} \in \operatorname{Arc}\left[\lambda_{0}, \lambda_{2}\right)$ and $\kappa>1$. Then there exists a positive integer $N \geq 0$ so that for any $\lambda \in \operatorname{Arc}\left[\lambda_{0}, \lambda^{\prime}\right]$, any $\omega \in \Omega$ and any $z \in J_{\omega, \lambda}$ we have

$$
\left|\left(\widehat{f}_{\omega, \lambda}^{N}\right)^{\prime}(z)\right| \geq \kappa .
$$

Proof. Let $N$ as in the previous lemma. For any $\lambda \in \mathbb{S}^{1}$, any $\omega \in \Omega$ and any $n \in \mathbb{N}$ the rational map $\widehat{f}_{\omega, \lambda}^{n}$ has no critical points on the unit circle. We may therefore find a constant 
$\varepsilon>0$ such that

$$
\left|\left(\widehat{f}_{\omega, \lambda}^{n}\right)^{\prime}(z)\right|>\varepsilon \quad \text { for all } \lambda, z \in \mathbb{S}^{1}, \text { for all } \omega \in \Omega, \text { for all } n \leq N .
$$

Let $j \in \mathbb{N}$ so that $\varepsilon \cdot \kappa^{j}>\kappa$.

Suppose now that $\lambda \in \operatorname{Arc}\left[\lambda_{0}, \lambda^{\prime}\right]$, that $\omega \in \Omega$ and that $z \in J_{\omega, \lambda}$. Thanks to the previous lemma there exist positive integers $J \geq j$ and $n_{1}, \ldots, n_{J} \in\{1, \ldots, N\}$ which satisfy $(j-1) \cdot N<n_{1}+\cdots+n_{J} \leq j N$ and so that the following assertion holds: if we write $m_{0}=0$ and $m_{i}=n_{1}+\cdots+n_{i}$ then for $i \in\{1, \ldots, J\}$,

$$
\left|\left(\widehat{f}_{\omega, \lambda}^{m_{i}, m_{i-1}}\right)^{\prime}\left(\widehat{f}_{\omega, \lambda}^{m_{i-1}}(z)\right)\right| \geq \kappa
$$

showing that

$$
\left|\left(\widehat{f}_{\omega, \lambda}^{j N}\right)^{\prime}(z)\right| \geq\left|\left(\widehat{f}_{\omega, \lambda}^{j N, j N-m_{j}}\right)^{\prime}\left(\widehat{f}_{\omega, \lambda}^{m_{j}}(z)\right)\right| \cdot \kappa^{J} \geq \varepsilon \cdot \kappa^{j} \geq \kappa .
$$

By choosing $j N$ instead of $N$, we conclude the proof of the proposition.

\subsection{Existence of expanding sequences.}

LEMMA 5.14. Given $d \in \mathbb{Z}_{\geq 2}, m \in\{1, \ldots, d-1\}, t \in(0,1)$ and $s \in(0, t)$, there exists $a k \in\{1, \ldots, d-1\}$ such that

$$
A_{k}:=\left(\frac{2 m-s}{d}\right) \cdot k+t
$$

is an element of the interval $(1,2)$ when reduced modulo 2.

Proof. Note that either $m<(d+s) / 2$ or $m>(d+s) / 2$, since $s \notin \mathbb{Z}$. We consider these two cases separately.

If $m<(d+s) / 2$ then $A_{k+1}-A_{k}<1$ for all $k$. It follows that for any open interval $(a, a+1)$ contained in the interval $\left[A_{0}, A_{d}\right]$, there exists an integer $0<k<d$ such that $A_{k} \in(a, a+1)$. Since $A_{0}=t<1$ and $A_{d}=2 m+t-s>2$, there exists a $k$ such that $A_{k} \in(1,2)$.

Now assume that $m>(d+s) / 2$ and define

$$
\tilde{A}_{p}=\left(\frac{2(d-m)+s}{d}\right) \cdot p+t-s .
$$

Observe that $A_{k}-\tilde{A}_{d-k}=2(k+m-d)$, and thus $A_{k} \equiv \tilde{A}_{d-k}(\bmod 2)$. Therefore it suffices to find a $p \in\{1, \ldots, d-1\}$ for which $\tilde{A}_{p} \in(1,2)$. Such a $p$ can be found by the same argument as above, because $\tilde{A}_{0}=t-s<1, \tilde{A}_{d}=2(d-m)+t>2$ and $\tilde{A}_{p+1}-$ $\tilde{A}_{p}<1$.

PROPOSITION 5.15. Let $d \geq 2$ and $\lambda \in \operatorname{Arc}\left[\lambda_{0}, \lambda_{1}\right)$. Then for every $z \in \mathbb{S}^{1} \backslash I$ there exists $\omega \in \Omega$ so that $z \in J_{\omega}$.

Proof. Choose $0<t<1$ so that $\lambda=e^{i \pi t}$. The function $f_{d}$ can be written as $f_{d}(z)=$ $\lambda \cdot \gamma(z)^{d}$, where $\gamma(z)=(z+b) /(b z+1)$ is a Möbius transformation that fixes the unit 
circle. It follows that we can find $d$ disjoint sets $J_{0}, \ldots, J_{d-1}$ such that

$$
f_{d}^{-1}(\operatorname{Arc}(1, \lambda))=J_{0} \cup \cdots \cup J_{d-1},
$$

ordered in such a way that for all $m \in\{0, \ldots, d-1\}$ we have

$$
\gamma\left(J_{m}\right)=\operatorname{Arc}\left(\exp \left(i \pi \frac{2 m-t}{d}\right), \exp \left(i \pi \frac{2 m}{d}\right)\right) .
$$

Since $f_{d}$ inverts the orientation of the unit circle and $f_{d}(1)=\lambda$, we have for $z \in \operatorname{Arc}(1, \lambda)$ close enough to 1 that $f_{d}(z) \in \operatorname{Arc}(1, \lambda)$. Furthermore, by Theorem 3.7 we cannot have $f_{d}(\lambda) \in \operatorname{Arc}(1, \lambda)$. This shows that one of the connected components of $f_{d}^{-1}(\operatorname{Arc}(1, \lambda))$ is of the form $J=\operatorname{Arc}\left(1, z^{\prime}\right) \subset \operatorname{Arc}(1, \lambda)$. This component must contain the arc $I$. Since $\gamma(1)=1$, we find that $J=J_{0}$.

Now let $J_{0}^{\prime}, \ldots, J_{d-1}^{\prime}$ denote the inverse arcs of $I$ under the map $f_{d}$ in such a way that $J_{m}^{\prime} \subset J_{m}$ for all $m$. Then we see that $J_{0}^{\prime}=I$. We now present a way to choose, given a $z \in \mathbb{S} \backslash I$, an integer $k$ such that $\widehat{f}_{k}(z) \in \mathbb{S} \backslash I$.

- If $z \notin f_{d}^{-1}(I)$, we can choose $k=d$ because then $\widehat{f}_{k}(z)=f_{d}(z) \notin I$.

- If $z \in f_{d}^{-1}(I)=J_{0}^{\prime} \cup \cdots \cup J_{d-1}^{\prime}$ but $z \notin I=J_{0}$, we see that $z \in J_{m}$ for some $m \in$ $\{1, \ldots, d-1\}$ and thus we can write

$$
\gamma(z)=\exp \left(i \pi \frac{2 m-s}{d}\right)
$$

for some $0<s<t$. We find that

$$
\widehat{f}_{k}(z)=f_{1}\left(\lambda \cdot \gamma(z)^{k}\right)=f_{1}\left(\exp \left(i \pi\left(\frac{2 m-s}{d} \cdot k+t\right)\right)\right) .
$$

According to Lemma 5.14, we can choose $k$ in such a way that $\lambda \cdot \gamma(z)^{k} \in \operatorname{Arc}(-1,1)$. Since $f_{1}(\operatorname{Arc}(-1,1))=\operatorname{Arc}(\lambda,-\lambda)$, we conclude that $\widehat{f}_{k}(z) \notin I$.

This procedure defines the first $n$ steps of the sequence $\omega$ and satisfies $\widehat{f}_{\omega}^{k}(z) \in \mathbb{S}^{1} \backslash I$ for all $k \leq n$. By iterating this procedure for the point $\widehat{f}_{\omega}^{n}(z)$ we find a sequence $\omega \in \Omega$ such that ${\widehat{f_{\omega}^{n}}}(z) \in \mathbb{S}^{1} \backslash I$ for all $n \in \mathbb{N}$. By (12) we conclude that $z \in J_{\omega}$.

Let $\lambda \in \operatorname{Arc}\left[\lambda_{0}, \lambda_{1}\right)$ and $z \in \operatorname{Arc}[\lambda, 1]$. Then we can modify the procedure described in the proof above to obtain the following proposition.

Proposition 5.16. Let $d \geq 2$ and $\lambda \in \operatorname{Arc}\left[\lambda_{0}, \lambda_{1}\right)$. Then for every $z \in \operatorname{Arc}[\lambda, 1]$ there exists $\omega \in \Omega$ so that $z \in J_{\omega}$ and

$$
\widehat{f}_{\omega}^{n}(z) \in \operatorname{Arc}[\lambda, 1] \text { for all } n \in \mathbb{N}
$$

COROllary 5.17. For every $\lambda \in \operatorname{Arc}\left[\lambda_{0}, \lambda_{2}\right)$ we have

$$
\widehat{\mathbb{C}} \backslash\left(\mathbb{S}^{1} \backslash I\right)=F_{\widehat{H}}, \quad \mathbb{S}^{1} \backslash I=J_{\widehat{H}} .
$$

Proof. Given $z \in \mathbb{S}^{1} \backslash I$, choose $\omega \in \Omega$ so that $z \in J_{\omega}$, which exists according to Proposition 5.15. For every positive integer $k \in \mathbb{N}$, by Proposition 5.13 we may find some $N_{k}$ such that

$$
\left|\left(\widehat{f}_{\omega}^{N_{k}}\right)^{\prime}(z)\right| \geq k
$$


showing that $\left|\left(\widehat{f}_{\omega}^{N_{k}}\right)^{\prime}(z)\right| \rightarrow \infty$ and therefore that $z \in J_{\widehat{H}}$. The two equalities follow from (10).

\section{Zeros for the semigroup}

Our goal in this section is to give a more precise description of the zeros of $Z_{G}(\lambda)$ in $\operatorname{Arc}\left[\lambda_{0}, \lambda_{1}\right]$ for trees $G$ that are spherically symmetric of bounded degree. We will prove case (3) of Theorem 1.4.

Theorem 6.1. Let $d \in \mathbb{N}_{\geq 2}$. Then there exists $\lambda_{3} \in \operatorname{Arc}\left(\lambda_{0},-1\right]$ so that the set of zero parameters for spherically symmetric trees of degree d contains a dense subset of $\operatorname{Arc}\left[\lambda_{0}, \lambda_{3}\right]$.

We will also prove the following weaker statement, albeit for a considerably larger circular arc.

THEOREM 6.2. Let $d \in \mathbb{N}_{\geq 2}$. Then the closure of the set of zero parameters of spherically symmetric trees of degree d contains $\operatorname{Arc}\left[\lambda_{0}, \lambda_{2}\right]$.

6.1. Proof of Theorem 6.1. Since we will repeatedly deal with distinct values of the parameter $\lambda$, we will always use the subscript $\lambda$ in order to specify the map $f_{\lambda}$ that we are using.

Choose a parameter value $\lambda^{\prime} \in \operatorname{Arc}\left(\lambda_{0}, \lambda_{2}\right)$. By Proposition 5.13 there exists a positive integer $N$ so that for every $\lambda \in \operatorname{Arc}\left[\lambda_{0}, \lambda^{\prime}\right]$, any $\omega \in \Omega$ and any $z \in J_{\omega, \lambda}$ we have

$$
\left|\left(\widehat{f}_{\omega, \lambda}^{N}\right)^{\prime}(z)\right|>3
$$

Having fixed $N$, it follows from the compactness of $\mathbb{S}^{1}$ that there exists a constant $C>0$ so that for any $z \in \mathbb{S}^{1}$ and any $\omega \in \Omega$ we have

$$
\left|\widehat{f}_{\omega, \lambda}^{N}(z)-\widehat{f}_{\omega, \mu}^{N}(z)\right| \leq C|\lambda-\mu| \text { for all } \lambda, \mu \in \mathbb{S}^{1}
$$

LEMma 6.3. There exist $\lambda_{3} \in \operatorname{Arc}\left[\lambda_{0}, \lambda^{\prime}\right]$ and a positive integer $M$ so that for every $\lambda \in$ $\operatorname{Arc}\left[\lambda_{0}, \lambda_{3}\right]$ and $1 \leq m \leq M$ we have

$$
f_{d, \lambda}^{m}(1) \in \operatorname{Arc}[\lambda, 1] \quad \text { and }\left|\partial_{\lambda} f_{d, \lambda}^{M}(1)\right|>2 C+1 .
$$

Proof. The point 1 is a repelling periodic point of order 2 for the map $f_{d, \lambda_{0}}$, meaning that $\left|\left(f_{d, \lambda_{0}}^{2}\right)^{\prime}(1)\right|>1$. Writing $\gamma(z)=(z+b) /(b z+1)$, we obtain

$$
\begin{aligned}
\left.\left(\partial_{\lambda} f_{d, \lambda}^{2 m}(1)\right)\right|_{\lambda=\lambda_{0}} & =\left.\left(\partial_{\lambda} f_{d, \lambda}^{2}(1)\right)\right|_{\lambda=\lambda_{0}}\left(1+\left(f_{d, \lambda_{0}}^{2}\right)^{\prime}(1)+\cdots+\left(f_{d, \lambda_{0}}^{2}\right)^{\prime}(1)^{m-1}\right) \\
& =\left(\gamma\left(\lambda_{0}\right)^{d}+f_{d, \lambda_{0}}^{\prime}(1)\right) \frac{\left(f_{d, \lambda_{0}}^{2}\right)^{\prime}(1)^{m}-1}{\left(f_{d, \lambda_{0}}^{2}\right)^{\prime}(1)-1}
\end{aligned}
$$


Observing that $\gamma\left(\lambda_{0}\right)^{d}+f_{d, \lambda_{0}}^{\prime}(1) \neq 0$, it follows that when $M=2 m$ for $m$ sufficiently large,

$$
\left|\left(\partial_{\lambda} f_{d, \lambda}^{M}(1)\right)\right|_{\lambda=\lambda_{0}} \mid>2 C+1
$$

By continuity there exists $\lambda_{3} \in \operatorname{Arc}\left[\lambda_{0}, \lambda^{\prime}\right]$ so that

$$
\left|\partial_{\lambda} f_{d, \lambda}^{M}(1)\right|>2 C+1 \quad \text { for all } \lambda \in \operatorname{Arc}\left[\lambda_{0}, \lambda_{3}\right] .
$$

Given $\lambda \in \operatorname{Arc}\left(\overline{\lambda_{1}}, \lambda_{1}\right)$, we write $z_{\lambda}, w_{\lambda}$ for the two boundary points of $I_{\lambda}$. These boundary points form a repelling 2-cycle, hence by the implicit function theorem they vary holomorphically in an open neighbourhood of $\operatorname{Arc}\left(\overline{\lambda_{1}}, \lambda_{1}\right)$. By exchanging the order of $z_{\lambda}$ and $w_{\lambda}$ if necessary, we may assume that $z_{\lambda_{0}}=1$. By Theorem 3.7, for every $\lambda \in \operatorname{Arc}\left(\lambda_{0}, \lambda_{1}\right)$ we have $z_{\lambda} \in \operatorname{Arc}(1, \lambda)$.

The point $f_{d, \lambda}(1)=\lambda$ clearly belongs to $\operatorname{Arc}[\lambda, 1]$. Recall that the map $f_{d, \lambda}^{2}$ preserves the orientation of the unit circle. Since $z_{\lambda}$ is a repelling fixed point, by replacing $\lambda_{3}$ with a parameter in $\operatorname{Arc}\left(\lambda_{0}, \lambda_{2}\right)$ sufficiently close to $\lambda_{0}$ so that the point $z_{\lambda}$ remains close to 1 for every $\lambda \in \operatorname{Arc}\left[\lambda_{0}, \lambda_{3}\right]$, we may assume that $f_{d, \lambda}^{2}(1) \in \operatorname{Arc}[\lambda, 1]$. Up to replacing at each step $\lambda_{3}$ with a parameter closer to $\lambda_{0}$, we may therefore assume that also $f_{d, \lambda}^{3}(1), \ldots, f_{d, \lambda}^{M}(1) \in \operatorname{Arc}[\lambda, 1]$, concluding the proof of the lemma.

By Proposition 4.2 we know that zero parameters $\lambda \in \overline{\mathcal{Z}_{\mathcal{C}_{d+1} \cap \mathbb{S}^{1}}}$ accumulate on $\lambda_{0}$. From now until the end of the proof of Theorem 6.1 we will fix the value of the parameter $\lambda \in \operatorname{Arc}\left(\lambda_{0}, \lambda_{3}\right]$.

By Theorem 3.7 we know that $I_{\lambda} \Subset \operatorname{Arc}(1, \lambda)$. This fact together with (14) and (13) implies the existence of the following constant.

Definition 6.4. (Choice of the constant $\varepsilon>0$ ) There exists a sufficiently small constant $\varepsilon>0$ so that the following three conditions are satisfied.

(a) The distance between the attracting interval $I_{\lambda}$ and $\operatorname{Arc}[\lambda, 1]$ is at least $\varepsilon$,

$$
\inf _{\substack{z \in I_{\lambda} \\ w \in \operatorname{Arc}[\lambda, 1]}}|z-w|>\varepsilon
$$

(b) For any $\mu \in B(\lambda, \varepsilon)$ we have

$$
\left|f_{d, \lambda}^{M}(1)-f_{d, \mu}^{M}(1)\right|>2 C|\lambda-\mu| .
$$

(c) For any $\omega \in \Omega$, any $z \in J_{\omega, \lambda}$ and any $w \in B(z, \varepsilon)$ we have

$$
\left|\widehat{f}_{\omega, \lambda}^{N}(z)-\widehat{f}_{\omega, \lambda}^{N}(w)\right|>2|z-w| \text {. }
$$

Definition 6.5. (Choice of the sequence $\sigma \in \Omega$ ) We define a sequence of the form

$$
\sigma=(\underbrace{d, \ldots, d}_{M}, \underbrace{\sigma_{M+1}, \sigma_{M+2}, \ldots}_{\sigma^{0}}),
$$

where $\sigma^{0} \in \Omega$ is chosen such that $\widehat{f}_{\sigma, \lambda}^{n}(1) \in \operatorname{Arc}[\lambda, 1]$ for all $n \in \mathbb{N}$. The existence of such a sequence is guaranteed by Proposition 5.16, and we have $1 \in J_{\sigma, \lambda}$. 
LEMMA 6.6. For every $\mu \in \mathbb{S}^{1} \backslash\{\lambda\}$ there exists a positive integer $n_{\mu}$ so that

$$
\left|\widehat{f}_{\sigma, \lambda}^{n_{\mu}}(1)-\widehat{f}_{\sigma, \mu}^{n_{\mu}}(1)\right| \geq \varepsilon .
$$

Proof. Suppose for the sake of contradiction that there exists $\mu \in \mathbb{S}^{1} \backslash\{\lambda\}$ so that $\left|\widehat{f}_{\omega, \lambda}^{n}(1)-\widehat{f}_{\omega, \mu}^{n}(1)\right|<\varepsilon$ for every $n \in \mathbb{N}$. Since $\widehat{f}_{\sigma, \mu}(1)=\mu$, it follows in particular that $|\lambda-\mu|<\varepsilon$. Therefore after the first $M$ steps we obtain that

$$
\left|\widehat{f}_{\sigma, \lambda}^{M}(1)-\widehat{f}_{\sigma, \mu}^{M}(1)\right|>2 C|\lambda-\mu| .
$$

Write $T: \Omega \rightarrow \Omega$ for the left shift map, and define $\sigma^{j}=T^{M+j N}(\sigma)$ and $z_{j, \mu}=$ $\widehat{f}_{\sigma, \mu}^{M+j N}(1) \in \mathbb{S}^{1}$. Thanks to the choice of the sequence $\sigma^{0}$, it follows that $z_{j, \lambda} \in \operatorname{Arc}[\lambda, 1]$ and that

$$
z_{j, \lambda} \in J_{\sigma^{j}, \lambda}
$$

We claim that for every $j \in \mathbb{N}$ we have $\left|z_{j, \lambda}-z_{j, \mu}\right|>\alpha_{n} C|\lambda-\mu|$, where $\alpha_{0}=2$ and $\alpha_{n+1}=2 \alpha_{n}-1$. The claim certainly holds for $j=0$. We will now assume it holds for $j \in \mathbb{N}$ and prove that it holds for $j+1$.

By the assumption on $\mu$ we know that $\left|z_{j, \lambda}-z_{j, \mu}\right|<\varepsilon$ for every $j \in \mathbb{N}$. Therefore, thanks to the choice of the constants $\varepsilon$ and $C$, we conclude that

$$
\begin{aligned}
\left|z_{j+1, \lambda}-z_{j+1, \mu}\right| & =\left|\widehat{f}_{\sigma, \lambda}^{M+(j+1) N}(1)-\widehat{f}_{\sigma, \mu}^{M+(j+1) N}(1)\right| \\
& >\left|\widehat{f}_{\sigma_{j}, \lambda}^{N}\left(z_{j, \lambda}\right)-\widehat{f}_{\sigma^{j}, \lambda}^{N}\left(z_{j, \mu}\right)\right|-\left|\widehat{f}_{\sigma^{j}, \lambda}^{N}\left(z_{j, \mu}\right)-\widehat{f}_{\sigma^{j}, \mu}^{N}\left(z_{j, \mu}\right)\right| \\
& >2\left|z_{j, \lambda}-z_{j, \mu}\right|-C|\lambda-\mu| \\
& >\left(2 \alpha_{n}-1\right) C|\lambda-\mu| .
\end{aligned}
$$

It then follows that for every $n$ we must have $\alpha_{n} C|\lambda-\mu|<\varepsilon$, which is possible only if $\lambda=\mu$, giving a contradiction.

LEMMA 6.7. There exists a positive integer $n_{0}$ so that every point in $\mathbb{S}^{1} \backslash I_{\lambda}$ lies at distance at most $\varepsilon / 4$ from the set

$$
\Sigma:=\bigcup_{\substack{\omega \in \Omega \\ 0 \leq n \leq n_{0}}} \widehat{f}_{\omega, \lambda}^{-n}(\{-1\}) .
$$

Proof. By Corollary 5.17 we have $\mathbb{S}^{1} \backslash I_{\lambda} \subset J_{\widehat{H}_{\lambda}}$. The semigroup $\hat{H}_{\lambda}$ has no exceptional points, therefore the collection of all preimages of a given point in $\widehat{\mathbb{C}}$ accumulates on the whole Julia set, and therefore on $\mathbb{S}^{1} \backslash I_{\lambda}$.

Writing $V=\bigcup_{g \in \widehat{H}_{\lambda}} g^{-1}(\{-1\})$, we therefore conclude that

$$
\mathbb{S}^{1} \backslash I_{\lambda} \subset \bar{V} \subset \bigcup_{z \in K} B(z, \varepsilon / 4) .
$$

By compactness of $\mathbb{S}^{1} \backslash I_{\lambda}$ we may find $w_{1}, \ldots, w_{\nu} \in V$ so that $B\left(w_{1}, \varepsilon / 4\right) \cup \cdots \cup$ $B\left(w_{n}, \varepsilon / 4\right)$ still covers the set $\mathbb{S}^{1} \backslash I_{\lambda}$. Every $w_{j}$ is the preimage of some element $g_{j} \in \widehat{H}_{\lambda}$ of length $n_{j}<\infty$, meaning that there exists $\omega_{j} \in \Omega$ so that

$$
\widehat{f}_{\omega_{j}, \lambda}^{n_{j}}\left(z_{j}\right)=-1 \text {. }
$$


By taking $n_{0}=\max n_{i}$ we obtain

$$
\left\{z_{1}, \ldots, z_{n}\right\} \subset \bigcup_{\substack{\omega \in \Omega \\ 0 \leq n \leq n_{0}}} \widehat{f}_{\omega, \lambda}^{-n}(\{-1\}),
$$

concluding the proof of the lemma.

Write $\Sigma=\left\{w_{1}, \ldots, w_{v}\right\}$. For every $w_{j} \in \Sigma$ there exist $\omega_{j} \in \Omega$ and $0 \leq n_{j} \leq n_{0}$ so that

$$
\widehat{f}_{\omega_{j}, \lambda}^{v_{j}}\left(w_{j}\right)=-1
$$

No element in the semigroup $\widehat{H}_{\lambda}$ can have critical points on the unit circle. Therefore by the implicit function theorem there exists a holomorphic map $\mu \mapsto w_{j, \mu}$ defined in a neighbourhood of $\lambda$ so that

$$
\widehat{f}_{\omega, \mu}^{n}\left(w_{j, \mu}\right)=-1, \quad w_{j, \lambda}=w_{j} .
$$

By taking $\delta_{0}>0$ sufficiently small we may assume that for every $j=1, \ldots, v$ the map $w_{j, \mu}$ is defined on $B\left(\lambda, \delta_{0}\right)$ and that

$$
\left|w_{j, \mu}-w_{j, \lambda}\right|<\varepsilon / 4 \text { for all } \mu \in B\left(\lambda, \delta_{0}\right) \text {, for all } j=1, \ldots, v .
$$

Given $\delta<\delta_{0}$, we choose $\mu^{\prime} \in \mathbb{S}^{1} \cap B(\lambda, \delta)^{*}$ in such a way that $\operatorname{Arc}\left[\lambda, \mu^{\prime}\right] \subset B(\lambda, \delta)$. We note that this last condition is not necessary, but simplifies the proof.

By Lemma 6.6 we may choose $n^{\prime}=n_{\mu^{\prime}}$ so that $\left|\widehat{f}_{\sigma, \lambda}^{n^{\prime}}(1)-\widehat{f}_{\sigma, \mu}^{n^{\prime}}(1)\right| \geq \varepsilon$. Given $\mu \in$ $\operatorname{Arc}\left[\lambda, \mu^{\prime}\right]$, write $z_{\mu}=\widehat{f}_{\sigma, \mu}^{n^{\prime}}(1)$.

Proposition 6.8. There exist $j \in\{1, \ldots, v\}$ and $\mu \in \operatorname{Arc}\left[\lambda, \mu^{\prime}\right]$ so that $z_{\mu}=w_{j, \mu}$.

Proof. By the definition of $\sigma$ we have that $z_{\lambda} \in \operatorname{Arc}[\lambda, 1]$, and by the choice of the constant $\varepsilon$ we know that $d\left(I_{\lambda}, z_{\lambda}\right)>\varepsilon$. Therefore, by replacing $\mu^{\prime}$ with another parameter closer to $\lambda$ in such a way that the value of $n^{\prime}$ does not change, we may further assume that $z_{\mu} \in \mathbb{S}^{1} \backslash I_{\lambda}$ for every $\mu \in \operatorname{Arc}\left[\lambda, \mu^{\prime}\right]$.

The image of the map $\operatorname{Arc}\left[\lambda, \mu^{\prime}\right] \ni \mu \mapsto z_{\mu}$ contains either $\operatorname{Arc}\left[z_{\lambda}, z_{\mu^{\prime}}\right]$ or $\operatorname{Arc}\left[z_{\mu^{\prime}}, z_{\lambda}\right]$ (notice that one of the two possibilities occurs, since the image does not intersect $I_{\lambda}$ ). We will prove the proposition assuming that the first case occurs; a similar proof works in the other case.

Choose a point $\zeta \in \operatorname{Arc}\left[z_{\lambda}, z_{\mu}\right]$ so that the distance of the point from both the extrema of the arc is greater than or equal to $\varepsilon / 2$, which is possible since $\left|z_{\lambda}-z_{\mu}\right| \geq \varepsilon$. Let $j \in$ $\{1, \ldots, \nu\}$ so that $\left|w_{j \lambda}-\zeta\right|<\varepsilon / 4$. It then follows that $w_{j, \lambda} \in \operatorname{Arc}\left[z_{\lambda}, z_{\mu^{\prime}}\right]$ and that

$$
\left|w_{j, \lambda}-z_{\lambda}\right|>\varepsilon / 4, \quad\left|w_{j, \lambda}-z_{\mu^{\prime}}\right|>\varepsilon / 4,
$$

and thanks to the fact that $\delta<\delta_{0}$ we conclude that

$$
w_{j, \mu} \in \operatorname{Arc}\left[z_{\lambda}, z_{\mu^{\prime}}\right] \text { for all } \mu \in \operatorname{Arc}\left[\lambda, \mu^{\prime}\right],
$$

and therefore that there exists $\mu \in \operatorname{Arc}\left[\lambda, \mu^{\prime}\right]$ so that $w_{j, \mu}=z_{\mu}$. 
Let $\mu \in \mathbb{S}^{1} \cap B(\lambda, \delta)$ and $j$ as in the previous lemma, and let $\omega_{j} \in \Omega$ and $n_{j} \in \mathbb{N}$ so that $\widehat{f}_{\omega_{j}, \mu}^{n_{j}}\left(w_{j, \mu}\right)=-1$. We conclude that

$$
\begin{aligned}
\widehat{f}_{\omega_{j}, \mu}^{n_{j}}\left(\widehat{f}_{\sigma, \mu}^{n^{\prime}}(1)\right) & =\widehat{f}_{\omega_{j}, \mu}^{n_{j}}\left(z_{\mu}\right) \\
& =\widehat{f}_{\omega_{j}, \mu}^{n_{j}}\left(w_{j, \mu}\right) \\
& =-1,
\end{aligned}
$$

concluding the proof of Theorem 6.1.

6.2. Proof of Theorem 6.2. Let $\lambda^{\prime} \in \operatorname{Arc}\left[\lambda_{0}, \lambda_{2}\right)$. By Proposition 5.13 there exists a positive integer $N \geq 0$ so that for any $\lambda \in \operatorname{Arc}\left[\lambda_{0}, \lambda^{\prime}\right]$ any $\omega \in \Omega$ and any $z \in J_{\omega, \lambda}$ we have

$$
\left|\left(\widehat{f}_{\omega, \lambda}^{N}\right)^{\prime}(z)\right|>3 .
$$

Once $N$ is fixed we have the following lemma.

LEMMA 6.9. There exist constants $\varepsilon, \delta>0$ so that for any $\lambda \in \operatorname{Arc}\left[\lambda_{0}, \lambda^{\prime}\right]$, any $\omega \in \Omega$, and any $z \in J_{\omega, \lambda}$, there exists a holomorphic map $F_{z, \omega, \lambda}: B(\lambda, \delta) \rightarrow B(z, \varepsilon)$ with $F_{z, \omega, \lambda}(\lambda)=z$, satisfying the following properties.

(1) $\left|\widehat{f}_{\omega, \lambda}^{k N}(z)-\widehat{f}_{\omega, \mu}^{k N}\left(F_{z, \omega, \lambda}(\mu)\right)\right|<\varepsilon$, for all $\mu \in B(\lambda, \delta)$, for all $k \geq 0$.

(2) Given any $\mu \in B(\lambda, \delta)$ and any $w \in B(z, \varepsilon) \backslash\left\{F_{z, \omega, \lambda}(\mu)\right\}$ there exists a positive integer $k$ so that

$$
\left|\widehat{f}_{\omega, \mu}^{k N}\left(F_{z, \omega, \lambda}(\mu)\right)-\widehat{f}_{\omega, \mu}^{k N}(w)\right| \geq 3 \varepsilon .
$$

Proof. Note that the second derivative of $\widehat{f}_{\omega, \lambda}^{N}$ is bounded in a neighbourhood of $\mathbb{S}^{1}$. It follows that for $\epsilon>0$ and $\delta>0$ sufficiently small, the maps $\widehat{f}_{\omega, \lambda}^{N}$ are uniformly expanding in a given neighbourhood of $J_{\omega, \lambda}$. The existence of the point $F_{z, \omega, \lambda}(\mu)$ follows immediately. The fact that $F_{z, \omega, \lambda}$ can be given as the limit of a sequence of contracting inverse branches implies the holomorphic dependency on $\mu$.

Proposition 6.10. Let $\lambda^{\prime} \in \operatorname{Arc}\left[\lambda_{0}, \lambda_{2}\right)$. Then the family of maps

$$
\mathcal{A}:=\left\{\lambda \mapsto g_{\lambda}(1) \mid g_{\lambda} \in \widehat{H}_{\lambda}\right\}
$$

is not normal near $\lambda^{\prime}$.

Proof. Let $\varepsilon, \delta>0$ as in the previous lemma. By Lemma 5.7 the map $\lambda \mapsto K_{\lambda}$ is upper semicontinuous in $\operatorname{Arc}\left[\lambda_{0}, \lambda_{2}\right)$, and $\lambda \mapsto I_{\lambda}$ is continuous. Therefore by compactness of $\operatorname{Arc}\left[\lambda_{0}, \lambda^{\prime}\right]$, and by taking smaller $\varepsilon, \delta$ if necessary, we may assume that

$$
\inf _{\substack{z \in K_{\lambda_{2}} \\ w \in \mathbb{S}^{1} \backslash I_{\lambda_{1}}}}|z-w|>2 \varepsilon .
$$

Now assume for the purpose of contradiction that the family of holomorphic functions $\mathcal{A}=\left\{\lambda \mapsto g_{\lambda}(1) \mid g_{\lambda} \in \widehat{H}_{\lambda}\right\}$ is normal near $\lambda^{\prime}$. By the Arzelá-Ascoli theorem it follows 
that there exists $\delta^{\prime}<\delta$ so that for every $\mu \in B\left(\lambda^{\prime}, \delta^{\prime}\right)$ we have

$$
\left|\widehat{f}_{\omega, \lambda^{\prime}}^{n}(1)-\widehat{f}_{\omega, \mu}^{n}(1)\right|<\varepsilon \quad \text { for all } \omega \in \Omega \text {, for all } n \in \mathbb{N} \text {. }
$$

By Proposition 5.16 we can fix $\omega \in \Omega$ so that $1 \in J_{\omega, \lambda}$ and $\widehat{f}_{\omega}^{n}(1) \in \operatorname{Arc}[\lambda, 1]$ for every positive integer $n$. It follows that $\left|\widehat{f}_{\omega, \lambda^{\prime}}^{k N}(1)-\widehat{f}_{\omega, \mu}^{k N}(1)\right|<\varepsilon$ on the ball $B\left(\lambda^{\prime}, \delta^{\prime}\right)$. By the identity principle it follows that $F_{1, \omega, \lambda^{\prime}}(\mu)=1$ on the bigger ball $B\left(\lambda^{\prime}, \delta\right)$, where $F_{1, \omega, \lambda^{\prime}}$ is the map defined in the previous lemma.

Now notice that, given $\lambda^{\prime \prime} \in B\left(\lambda^{\prime}, \delta\right) \cap \operatorname{Arc}\left[\lambda_{0}, \lambda^{\prime}\right]$, we have $1 \in J_{\omega, \lambda^{\prime \prime}}$. If this were not the case then by (10) we could find a positive integer $n$ so that $\widehat{f}_{\omega, \lambda^{\prime \prime}}(1) \in I_{\lambda^{\prime \prime}} \subset F_{\widehat{\mathcal{G}}}$, and by Proposition 5.8 we conclude that $\widehat{f}_{\omega, \lambda^{\prime \prime}}^{n}(1) \rightarrow K_{\lambda}^{\prime \prime}$. In particular, when $k$ is sufficiently large the point $\widehat{f}_{\omega, \lambda^{\prime \prime}}^{k N}(1)$ lies at distance strictly less than $\varepsilon$ from the set $K_{\lambda^{\prime \prime}}$. Since instead the point $\widehat{f}_{\omega, \lambda^{\prime}}^{k N}$ (1) lies in $\mathbb{S}^{1} \backslash I_{\lambda^{\prime}}$ and the two sets $\mathbb{S}^{1} \backslash I_{\lambda^{\prime}}$ and $K_{\lambda^{\prime \prime}}$ have distance greater than $2 \varepsilon$, we conclude that for $k$ sufficiently large,

$$
\left|\widehat{f}_{\omega, \lambda^{\prime}}^{k N}(1)-\widehat{f}_{\omega, \lambda^{\prime \prime}}^{k N}(1)\right| \geq \varepsilon,
$$

contradicting the fact that $F_{1, \omega, \lambda^{\prime}}\left(\lambda^{\prime \prime}\right)=1$.

On the intersection $U=B\left(\lambda^{\prime}, \delta\right) \cap B\left(\lambda^{\prime \prime}, \delta\right)$ the maps $F_{1}=F_{1, \omega, \lambda^{\prime}}$ and $F_{2}=F_{1, \omega, \lambda^{\prime \prime}}$ are well defined. For every $\mu \in U$ and every positive integer $k$ we have $F_{1}(\mu)=1$ and

$$
\begin{array}{r}
\left|\widehat{f}_{\omega, \lambda^{\prime}}^{k N}(1)-\widehat{f}_{\omega, \mu}^{k N}(1)\right|<\varepsilon, \\
\left|\widehat{f}_{\omega, \lambda^{\prime}}^{k N}(1)-\widehat{f}_{\omega, \lambda^{\prime \prime}}^{k N}(1)\right|<\varepsilon, \\
\left|\widehat{f}_{\omega, \lambda^{\prime \prime}}^{k N}(1)-\widehat{f}_{\omega, \mu}^{k N}\left(F_{2}(\mu)\right)\right|<\varepsilon,
\end{array}
$$

which implies that

$$
\left|\widehat{f}_{\omega, \mu}^{k N}(1)-\widehat{f}_{\omega, \mu}^{k N}\left(F_{2}(\mu)\right)\right|<3 \varepsilon \quad \text { for all } k \in \mathbb{N},
$$

and thus that $F_{2}(\mu)=1$ on the open set $U$. By the identity principle it follows that $F_{1, \omega, \lambda^{\prime \prime}}(\mu)=1$ on $B\left(\lambda^{\prime \prime}, \delta\right)$. By iterating this procedure we conclude that for every $\lambda \in$ $\operatorname{Arc}\left[\lambda_{0}, \lambda^{\prime}\right]$ we have $1 \in J_{\omega, \lambda}$ and $F_{1, \omega, \lambda}(\mu)=1$ on the all ball $B(\lambda, \delta)$. It follows that $1 \in J_{\omega, \lambda_{0}}$.

Recall that by Theorem 3.7 we have $I_{\lambda_{0}}=\operatorname{Arc}\left(1, \lambda_{0}\right)$, therefore by Lemma 5.5 we have $1 \in J_{\omega, \lambda_{0}}$ if and only if $\omega=(d, d, d, \ldots)$. Hence for every $\lambda \in \operatorname{Arc}\left[\lambda_{0}, \lambda^{\prime}\right]$ we must have

$$
f_{d, \lambda}^{n}(1) \in \mathbb{S}^{1} \backslash I_{\lambda},
$$

which implies that $1 \in J_{d, \lambda}$ for all $\lambda \in \operatorname{Arc}\left[\lambda_{0}, \lambda^{\prime}\right]$, which contradicts Lemma 3.9. This concludes the proof of the proposition.

Theorem 6.2 now follows from Montel's theorem as in Lemma 2.7.

Acknowledgements. The research leading to these results has received funding from the European Research Council under the European Union's Seventh Framework Programme (FP7/2007-2013) / ERC grant agreement no. 617747. This work was supported by NWO TOP grant 613.001.851 and NWO TOP grant 614.001.506. 


\section{REFERENCES}

[Bar16] A. Barvinok. Combinatorics and Complexity of Partition Functions (Algorithms and Combinatorics, 30). Springer, Cham, 2016.

[BG01] J. C. A. Barata and P. S. Goldbaum. On the distribution and gap structure of Lee-Yang zeros for the Ising model: periodic and aperiodic couplings. J. Stat. Phys. 103(5/6) (2001), 857-891.

[BM97] J. C. A. Barata and D. H. U. Marchetti. Griffiths' singularities in diluted Ising models on the Cayley tree. J. Stat. Phys. 88(1-2), (1997), 231-268.

[CHJR19] I. Chio, C. He, A. L. Ji and R. K. W. Roeder. Limiting measure of Lee-Yang zeros for the Cayley tree. Comm. Math. Phys. 370(3) (2019), 925-957.

[DF08] R. Dujardin and C. Favre. Distribution of rational maps with a preperiodic critical point. Amer. J. Math. 130(4) (2008), 979-1032.

[Lev81] G. M. Levin. Irregular values of the parameter of a family of polynomial mappings. Uspekhi Mat. Nauk 36(6(222)) (1981), 219-220.

[LY52a] T. D. Lee and C. N. Yang. Statistical theory of equations of state and phase transitions. I. Theory of condensation. Phys. Rev. 87(2) (1952), 404-409.

[LY52b] T. D. Lee and C. N. Yang. Statistical theory of equations of state and phase transitions. II. Lattice gas and Ising model. Phys. Rev. 87(2) (1952), 410-419.

[Lyu83] M. Y. Lyubich. Some typical properties of the dynamics of rational mappings. Uspekhi Mat. Nauk 38(5(233)) (1983), 197-198.

[McM00] C. T. McMullen. The Mandelbrot set is universal. The Mandelbrot Set, Theme and Variations (London Mathematical Society Lecture Note Series, 274). Cambridge University Press, Cambridge, 2000, pp. $1-17$.

[McM94] C. T. McMullen. Complex Dynamics and Renormalization (Annals of Mathematics Studies, 135). Princeton University Press, Princeton, NJ, 1994.

[MH77] E. Müller-Hartmann. Theory of the Ising model on a Cayley tree. Z. Phys. B Condens. Matter 27(2) (1977), 161-168.

[MHZ75] E. Müller-Hartmann and J. Zittartz. Phase transitions of continuous order: Ising model on a Cayley tree. Z. Phys. B Condens. Matter 22(1) (1975), 59-67.

[Mil00] J. Milnor. On rational maps with two critical points. Exp. Math. 9(4) (2000), 481-522.

[MnSS83] R. Mañé, P. Sad and D. Sullivan. On the dynamics of rational maps. Ann. Sci. Éc. Norm. Supér. (4) 16(2) (1983), 193-217.

[PR17] V. Patel and G. Regts. Deterministic polynomial-time approximation algorithms for partition functions and graph polynomials. SIAM J. Comput. 46(6) (2017), 1893-1919.

[PR19] H. Peters and G. Regts. On a conjecture of Sokal concerning roots of the independence polynomial. Michigan Math. J. 68(1) (2019), 33-35.

[PR20] H. Peters and G. Regts. Location of zeros for the partition function of the Ising model on bounded degree graphs. J. Lond. Math. Soc. (2) 101(2) (2020), 795-785.

[Slo91] Z. Slodkowski. Holomorphic motions and polynomial hulls. Proc. Amer. Math. Soc. 111(2) (1991), 347-355.

[Sum97] H. Sumi. On dynamics of hyperbolic rational semigroups. J. Math. Kyoto Univ. 37(4) (1997), 717-733.

[Sum98] H. Sumi. On Hausdorff dimension of Julia sets of hyperbolic rational semigroups. Kodai Math. J. 21(1) (1998), 10-28. 